\title{
Domain growth morphology in curvature-driven two-dimensional coarsening
}

\author{
Alberto Sicilia, ${ }^{1}$ Jeferson J. Arenzon, ${ }^{2}$ Alan J. Bray, ${ }^{3}$ and Leticia F. Cugliandolo ${ }^{1}$ \\ ${ }^{1}$ Université Pierre et Marie Curie_Paris VI, LPTHE UMR 7589, 4 Place Jussieu, 75252 Paris Cedex 05, France \\ ${ }^{2}$ Instituto de Física, Universidade Federal do Rio Grande do Sul, CP 15051, 91501-970 Porto Alegre RS, Brazil \\ ${ }^{3}$ School of Physics and Astronomy, University of Manchester, Manchester M13 9PL, United Kingdom
}

(Received 14 August 2007; published 17 December 2007)

\begin{abstract}
We study the distribution of domain areas, areas enclosed by domain boundaries ("hulls"), and perimeters for curvature-driven two-dimensional coarsening, employing a combination of exact analysis and numerical studies, for various initial conditions. We show that the number of hulls per unit area, $n_{h}(A, t) d A$, with enclosed area in the interval $(A, A+d A)$, is described, for a disordered initial condition, by the scaling function $n_{h}(A, t)=2 c_{h} /\left(A+\lambda_{h} t\right)^{2}$, where $c_{h}=1 / 8 \pi \sqrt{3} \approx 0.023$ is a universal constant and $\lambda_{h}$ is a material parameter. For a critical initial condition, the same form is obtained, with the same $\lambda_{h}$ but with $c_{h}$ replaced by $c_{h} / 2$. For the distribution of domain areas, we argue that the corresponding scaling function has, for random initial conditions, the form $n_{d}(A, t)=2 c_{d}\left(\lambda_{d} t\right)^{\tau^{\prime}-2} /\left(A+\lambda_{d} t\right)^{\tau^{\prime}}$, where $c_{d}$ and $\lambda_{d}$ are numerically very close to $c_{h}$ and $\lambda_{h}$, respectively, and $\tau^{\prime}=187 / 91 \approx 2.055$. For critical initial conditions, one replaces $c_{d}$ by $c_{d} / 2$ and the exponent is $\tau=379 / 187 \approx 2.027$. These results are extended to describe the number density of the length of hulls and domain walls surrounding connected clusters of aligned spins. These predictions are supported by extensive numerical simulations. We also study numerically the geometric properties of the boundaries and areas.
\end{abstract}

DOI: 10.1103/PhysRevE.76.061116

PACS number(s): 64.60.Cn, 68.43.Jk

\section{INTRODUCTION}

In this work we obtain some exact results for the coarsening dynamics [1] of a nonconserved scalar field in two dimensions, demonstrating, en passant, the validity of the dynamical scaling hypothesis for this system. We study the morphology of the domain structure, which is illustrated in Fig. 1 for the coarsening of the two-dimensional Ising model (2dIM) on a square lattice quenched from an equilibrium state at $T_{0}>T_{c}$.

A domain is a region of connected aligned spins. Each domain has one external perimeter which is called the hull. The hull enclosed area is the total area within this perimeter, i.e., the domain area plus the area of any internal subdomain. The domain perimeter is the total length of the interface between the chosen domain and the neighboring onesincluding the hull and internal borders. See Fig. 2 for a sketch explaining these definitions.

The paper deals primarily with the distributions of two characteristic areas, the domain area and the hull enclosed area, and their associated lengths, the domain wall perimeter and the hull length.

Naively, one may imagine that coarsening is basically due to the coalescence of small domains that form larger ones. However, in two-dimensional curvature-driven coarsening coalescence processes are quite unimportant as shown by the Allen-Cahn result. All the interfaces move with a local velocity that is proportional to the local curvature and points in the direction of decreasing the curvature; therefore, interfaces tend to disappear independently of one another. This is the reason why we first focused on the statistics of hull enclosed areas, quantities that depend on the motion of a single and connected interface, and not on the statistics of the more natural domain areas. Next we expressed the statistics of the domain areas in terms of the simpler and more clear statistics of hull enclosed areas.
Hull enclosed and domain areas have distributions that, at late times after the quench into the ordered phase, exhibit, according to the scaling hypothesis, the scaling form $n(A, t)=t^{-2} f(A / t)$, where $n(A, t) d A$ is the number of hulls (domains) per unit area with area in the range $(A, A+d A)$. The argument of the scaling function arises from the fact that the characteristic length scale is known to grow as $t^{1 / 2}$, so the characteristic area (of hulls and domains) grows as $t$. The scaling function $f(x)$ will be different for domains and hulls. The prefactor $t^{-2}$ follows from the fact that there is of order one hull (or domain) per scale area. Our analytical result is an elegant application of the Gauss-Bonnet theorem and, it should be emphasized, its simplicity relies on the dimensionality of the system being 2 . Indeed, in three dimensions, the time variation of the hull enclosed volume depends on a characteristic size of the domain. A similar dependence on the dimension is also observed in the von Neumann's law for cellular systems, whose simple form, independent of any linear size of the system, is also only observed in two dimensions $[3,4]$.

In this paper we derive these scaling forms from first principles (i.e., without recourse to the scaling hypothesis), and determine explicitly the scaling functions. Some of our results have appeared earlier [5].

Hulls and domain boundary lengths are themselves distributed quantities related in a nontrivial manner to their corresponding areas. In this paper we examine the geometry of these structures and we derive the number density of hull and domain wall lengths showing that these distributions also satisfy scaling.

The organization of the paper is as follows. In Sec. II we recall known results about the equilibrium distribution of hull enclosed and domain areas at critical percolation and critical Ising initial conditions. We also summarize known results about the equilibrium distribution of domain walls and hulls and their geometrical relation to their associated 


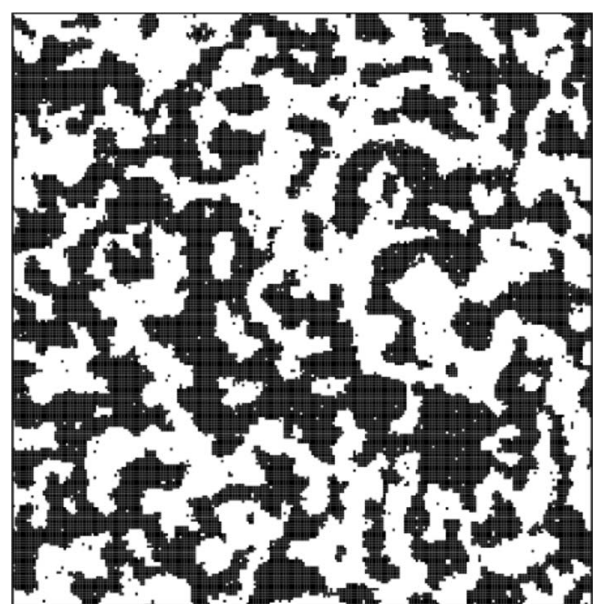

(a)

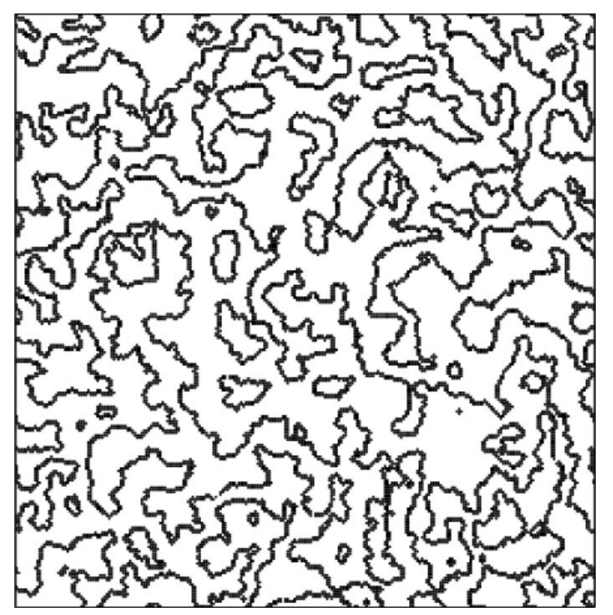

(b)

FIG. 1. Snapshots of the 2D Ising model at time $t=32$ Monte Carlo steps (MCs) after a quench from infinite temperature, $T_{0}$ $\rightarrow \infty$, to the working temperature $T=1.5 \simeq 0.66 T_{c}$. (a) We show the raw data, where the domain structure as well as the thermal fluctuations within the domains are visible. (b) We show the boundaries between regions of opposite sign in the configuration to the left, using a variant of the algorithm in Ref. [2] to make the domain structure clearer.

areas. In Sec. III we derive some generic results that stem from a number of sum rules and the use of the scaling hypothesis. In Sec. IV we explain the analytic derivation of the time-dependent hull enclosed and domain area distributions. These arguments do not rely on any scaling hypothesis but rather demonstrate its validity. In Sec. $\mathrm{V}$ we show our numerical results for the statistics of areas in the 2dIM evolving with Monte Carlo dynamics. Section VI is devoted to the analysis, both analytical and numerical, of the geometry of hulls and domain walls during the dynamics and their relation to their corresponding areas. Finally, in the Conclusion we discuss future studies along these lines. We also add two appendixes in which we describe the algorithm used to identify and count the hull enclosed areas, and for the sake of comparison we present the distribution of domain lengths in the one-dimensional Ising model.

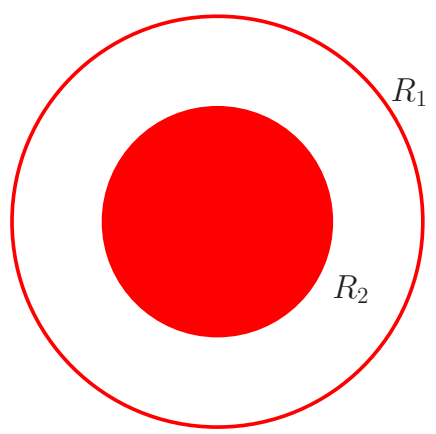

FIG. 2. (Color online) A sketch of a configuration with two concentric and circular interfaces with radius $R_{1}$ and $R_{2}$ is shown to illustrate the definition of hull enclosed and domain areas as well as hull and domain-wall perimeters. This configuration has two hull enclosed areas, $A_{h}^{(1)}=\pi R_{1}^{2}$ and $A_{h}^{(2)}=\pi R_{2}^{2}$, and two domains with areas $A_{d}^{(1)}=\pi\left(R_{1}^{2}-R_{2}^{2}\right)$ and $A_{d}^{(2)}=\pi R_{2}^{2}$. There are two hulls with length $p_{h}^{(1)}=2 \pi R_{1}$ and $p_{h}^{(2)}=2 \pi R_{2}$, and two domain walls with length $p_{d}^{(1)}=2 \pi\left(R_{1}+R_{2}\right)$ and $p_{d}^{(2)}=2 \pi R_{2}$.

\section{EQUILIBRIUM DISTRIBUTIONS}

In this section we summarize known results on the equilibrium hull enclosed and domain area distributions as well as the number density of perimeters, both at critical percolation and critical Ising conditions in two dimensions, see Fig. 2. These will act as initial conditions for the coarsening dynamics.

\section{A. Area distributions}

A hull enclosed area is defined as the full interior of a domain boundary-irrespective of there being other interfaces and thus regions of the opposite phase within. The equilibrium hull enclosed area distributions at percolating criticality and Ising criticality have been computed by Cardy and Ziff [6] in two dimensions:

$$
n_{h}(A, 0) \sim\left\{\begin{array}{rr}
2 c_{h} / A^{2}, & \text { critical percolation } \\
c_{h} / A^{2}, & \text { critical Ising. }
\end{array}\right.
$$

These results are valid for $A_{0} \ll A \ll L^{2}$, with $A_{0}$ a microscopic area and $L^{2}$ the system size. Note also that we are taking an extra factor 2 arising from the fact that there are two types of hull enclosed areas, corresponding to the two phases, while the Cardy-Ziff results accounts only for clusters of occupied sites (and not clusters of unoccupied sites). $n_{h}(A, 0) d A$ is the number density of hulls per unit area with enclosed area in the interval $(A, A+d A)$ (we keep the notation to be used later and set $t=0)$. The adimensional constant $c_{h}$ is a universal quantity that takes a very small value: $c_{h}$ $=1 /(8 \pi \sqrt{3}) \approx 0.022972$. The smallness of $c_{h}$ plays an important role in the analysis of Sec. IV.

The distribution of domain areas-we recall that domains are clusters of connected aligned spins - at critical percolation is given by [7] 


$$
n_{d}(A, 0) \sim \frac{2 c_{d} A_{0}^{\tau^{\prime}-2}}{A^{\tau^{\prime}}}, \quad \text { with } \tau^{\prime}=\frac{187}{91} \approx 2.055
$$

Of course, the constants $c_{d}$ and $A_{0}$ are not separately defined by this relationship, only the ratio $c_{d} A_{0}^{\tau^{\prime}-2}$. In practice it is convenient to choose $c_{d}$ to be the value appearing in the domain area distribution at general times-see Eq. (9). The quantity $A_{0}$ can be interpreted as a microscopic area, and will be introduced at various points as a small-area cutoff. The quantity $A_{0}^{\tau^{\prime}-2}$ in Eq. (2) sets the units in such a way that $\left[n_{d}\right]=A_{0}^{-2}$. This result is also valid in the limit $A_{0} \ll A \ll L^{2}$.

In equilibrium at $T_{c}$, Stella and Vanderzande [8] computed the number density of domains with area $A$,

$$
n_{d}(A, 0) \sim \frac{c_{d} A_{0}^{\tau-2}}{A^{\tau}}, \quad \text { with } \tau=\frac{379}{187} \approx 2.027
$$

in the large $A$ limit. Janke and Schakel [9] confirmed this claim numerically finding $\tau \approx 2.0269$. Motivated by the Cardy-Ziff result for hull enclosed areas, we conjecture that the prefactor $c_{d}$ in Eq. (3) is the same $c_{d}$ (up to terms of order $c_{h}^{2}$ ) as that appearing in the prefactor $2 c_{d}$ for critical percolation. We discuss this point in detail at the beginning of Sec. IV B, and we check this hypothesis numerically in Sec. V.

We find it useful to include the small-area cutoff, $A_{0}$, in these number densities, transforming the denominators to $\left(A+A_{0}\right)^{2}$ or $\left(A+A_{0}\right)^{\tau, \tau^{\prime}}$ for hull enclosed and domain areas, respectively.

\section{B. Perimeter distributions}

One can equally study the length of hulls (external perimeters) and domain walls (including external and internal boundaries).

The number density $n_{h}(p, 0)$ of hulls with length $p$, defined as the number of spins on the hull, at the critical point of the 2dIM was computed by Vanderzande and Stella [10],

$$
n_{h}(p, 0) \sim \frac{c_{p_{h}} p_{0}^{\zeta_{h}-3}}{\left(p+p_{0}\right)^{\zeta_{h}}}, \quad \text { with } \zeta_{h}=\frac{27}{11} \approx 2.454
$$

The value of the constant $c_{p_{h}}$ was not estimated. As far as we know there is no analytic prediction for the number density of domain walls at critical Ising conditions that should have the same functional form though with a possibly different exponent $\zeta_{d}$ and a different constant $c_{p_{d}}$. $p_{0}$ is a microscopic length that we define as $p_{0}^{2}=A_{0}$.

For critical percolation, the number density of hulls with given perimeter was obtained by Saleur and Duplantier [11],

$$
n_{h}(p, 0) \sim \frac{c_{p_{h}}^{\prime} p_{0}^{\prime \zeta_{h}^{\prime}-3}}{\left(p+p_{0}\right)^{\zeta_{h}^{\prime}}}, \quad \text { with } \zeta_{h}^{\prime}=\frac{15}{7} \approx 2.143,
$$

compatible with the numerical study [12]. Again, the numerical value of the constant $c_{p_{h}}^{\prime}$ is not known. As far as we know, the analog of Eq. (5) for domain walls at critical percolation is not known, though we expect the same functional form with different constant $c_{p_{d}}^{\prime}$ and exponent $\zeta_{d}^{\prime}$.
In Sec. VI we show numerical results for the equilibrium perimeter length number densities of critical Ising and infinite temperature configurations.

\section{Fractal properties}

Several authors studied the fractal properties of areas and perimeters in critical Ising and critical percolation equilibrium conditions using different analytic methods that include conformal invariance and renormalization group and Coulomb gas techniques $[8,10,11,13,14]$. Many numerical studies $[9,12,15-17]$ confirmed and complemented the results in these analytic works. These works focused on the fractal dimensions of the domain area, $D_{d}$, and of the hull length, $D_{h}$. In two dimensions these exponents are linked to the distribution exponents $\tau$ and $\zeta_{h}$ as [7]

$$
D_{d}=\frac{2}{\tau-1}, \quad D_{h}=\frac{2}{\zeta_{h}-1},
$$

and similarly for the primed quantities.

We concentrate on the fractal properties of geometric structures by comparing the area of the clusters to their associated perimeter. This approach was used by Cambier and Nauenberg [18], who studied domain walls in the 2dIMwith internal and external border-at equilibrium below, but near $T_{c}$, and found

$$
A \sim p^{1.43} .
$$

The proportionality constant is not given. In Sec. VI we revisit the geometric properties of the clusters at $T_{c}$ and the corresponding one at very high temperature as well as their zero temperature evolution.

\section{SOME GENERAL RESULTS}

A number of general properties of hull enclosed and domain areas as well as hull and domain wall perimeters can be easily derived just by using the scaling hypothesis and two sum rules. We summarize them here.

\section{A. Scaling}

At long times, and irrespective of the initial condition, the total number of domain and hull enclosed areas per unit area, $N_{d, h}(t)=\int_{0}^{\infty} d A n_{d, h}(A, t)$, should scale as $R^{-d}(t)$ in $d$ dimensions, with $R(t)$ a characteristic length scale usually associated to the "typical" domain radius. For pure ferromagnetic coarsening, $R(t) \sim\left(\lambda_{d} t\right)^{1 / 2}$ from which it follows that $N_{d, h}(t)$ $\sim t^{-1}$ in $d=2$. Since characteristic areas scale as $R^{2}(t) \sim t$, the scaling hypothesis implies that the domain and hull enclosed area distributions have the forms $n_{d, h}(A, t)=t^{-2} f_{d, h}(A / t)$.

In Sec. IV we present arguments that the initial distributions of hull enclosed and domain areas determine the forms of these distributions at late times. In particular, the initial forms are modified in a rather simple way at later times, such that the scaling forms are already suggested by the initial conditions. In the following we outline the consequences of this line of argument and defer detailed analysis to Sec. IV. 
Let us first discuss domains with critical Ising initial conditions. Retaining the initial form (3), but including an extra time-dependent factor to satisfy the desired scaling form at large times, the expression for $n_{d}(A, t)$ is

$$
n_{d}(A, t) \sim \frac{c_{d}\left[\lambda_{d}\left(t+t_{0}\right)\right]^{\tau-2}}{\left[A+\lambda_{d}\left(t+t_{0}\right)\right]^{\tau}}
$$

at long times. Here, $t_{0}$ is a microscopic time, and $\lambda_{d}$ is a phenomenological parameter with the dimensions of a diffusion constant. Setting $t=0$ one recovers Eq. (3) provided $A_{0}=\lambda_{d} t_{0}$ and $A \gg A_{0}$. Indeed, $t_{0}$ is defined through this requirement while $A_{0}$ is the by now usual microscopic area. For large $t, t_{0}$ can be neglected and the conventional scaling form is recovered.

For infinite temperature initial conditions we propose

$$
n_{d}(A, t) \sim \frac{2 c_{d}\left[\lambda_{d}\left(t+t_{0}\right)\right]^{\tau^{\prime}-2}}{\left[A+\lambda_{d}\left(t+t_{0}\right)\right]^{\tau^{\prime}}}
$$

that also satisfies the scaling form asymptotically.

One can easily check that the obvious generalization of Eq. (1) including time

$$
n_{h}(A, t) \sim \frac{(2) c_{h}}{\left(A+\lambda_{h} t\right)^{2}}
$$

also has the desired scaling form. The constant $\lambda_{h}$ is another phenomenological parameter. Its value will turn out to be very close to $\lambda_{d}$. The factor 2 in the brackets in Eq. (10) takes into account the two types of initial condition.

We remark that in Eq. (10) we do not explicitly include a short-time cutoff $t_{0}$, while it is necessary in Eqs. (8) and (9) to connect smoothly to the initial condition.

Scaling can also be used to predict the time dependence of the number density of hull and domain wall lengths. It yields

$$
n_{d, h}(p, t) \sim \frac{c_{p_{d}, p_{h}}\left[\left(\lambda_{d, h} t\right)^{1 / 2}+p_{0}\right]^{\zeta_{d, h}-3}}{\left[p+\left(\lambda_{d, h} t\right)^{1 / 2}+p_{0}\right]^{\zeta_{d, h}}}
$$

with the constant and exponent values depending on the initial condition and whether we are studying hulls or domain walls. This form is based on the assumption that the characteristic perimeters evolve in time as $\left(\lambda_{d, h} t\right)^{1 / 2}$.

\section{B. Sum rules}

We now present two exact sum rules which provide useful input for the analysis of $n_{d, h}(A, t)$. These sum rules apply at all times $t \geqslant 0$, for any initial condition, and for any working temperature.

The first sum rule follows from the fact that the total domain area, per unit area of the system, is unity since each space point (or lattice site) belongs to one and only one domain. This gives

$$
\int_{0}^{\infty} d A A n_{d}(A, t)=1
$$

The second sum rule follows from the fact that the total number of hull enclosed areas, $N_{h}(t)$, is equal to the total number of domains, $N_{d}(t)$, since each domain can be associated with a unique hull, namely the hull that forms its external boundary. This yields

$$
\begin{aligned}
N_{d}(t) & \equiv \int_{0}^{\infty} d A n_{d}(A, t) \\
& =\int_{0}^{\infty} d A n_{h}(A, t) \equiv N_{h}(t) .
\end{aligned}
$$

Equations (1) and (2) and their generalization describing the time dependence, have been shown to hold for large areas $\left(A \gg A_{0}\right)$ only and the number density can take a different form at small values of $A$. From the constraints (12) and (13), using Eqs. (8)-(10), we shall derive approximate relations between the constants $c_{d}, c_{h}, \lambda_{d}, \lambda_{h}, \tau$, and $\tau^{\prime}$ expected to hold at any working temperature $T$. These relations are exact to first order in the small quantity $c_{h}$.

For the number densities of hulls or domain walls with given perimeter we have only one sum rule. The total number density must equal the number density of domains and hull enclosed areas:

$$
N_{p_{d}, p_{h}}(t) \equiv \int_{0}^{\infty} d p n_{d, h}(p, t)=N_{d}(t)=N_{h}(t)
$$

\section{Critical Ising initial conditions}

The constraint on the total area (12) using Eq. (8) for the number density of domain areas yields

$$
c_{d}=(\tau-2)(\tau-1) \approx 0.02745
$$

where the numerical value was obtained for $\tau=379 / 187$. If one takes into account that the minimal area is $A_{0}$ (and not 0 ) the result is slightly different and it varies, though very weakly, with $t$. Indeed, the value of $c_{d}$ decreases from $c_{d}$ $=(\tau-2)(\tau-1) 2^{\tau-1} / \tau$ at $t=0$ to $c_{d}=(\tau-2)(\tau-1)$ at $t \gg t_{0}$ $=A_{0} / \lambda_{d}$. Inserting $\tau=379 / 187$ gives

$$
0.02745 \leqslant c_{d} \leqslant 0.02760,
$$

a rather narrow interval. Note that $c_{d}$ computed using Eq. (15) is quite close to the analytical result for $c_{h}$, namely $c_{h}^{\text {an }}$ $=1 / 8 \pi \sqrt{3} \sim 0.02297$. Indeed, $c_{d}-c_{h}^{\text {an }} \approx 0.00448$ (which is order $\left.c_{h}^{\mathrm{an}} / 5\right)$ where we used the minimum value of $c_{d}$ and the analytical value of $c_{h}$.

We now consider the sum rule (13), at times $t=0$ and $t$ $\gg A_{0} / \lambda_{h}$, distinguishing the integral over the full interval $[0, \infty)$ from the one that takes into account the finite minimal area $\left[A_{0}, \infty\right)$. We denote by $A_{1}$ the lower limit of the integration interval. We also include a small-area cutoff $A_{0}$ in the hull enclosed area number density, $n_{h}(A, 0) \sim c_{h} /\left(A+A_{0}\right)^{2}$.

At time $t=0$ we find

$$
c_{d}=c_{h}(\tau-1) \frac{\left(A_{1}+A_{0}\right)^{\tau-2}}{A_{0}^{\tau-2}} .
$$

Using now the expressions for $c_{d}$ derived above we relate $c_{h}$ to $\tau$ : 


$$
c_{h}=\left\{\begin{array}{cc}
(\tau-2) \approx 0.02674, & A_{1}=0, \\
\frac{2(\tau-2)}{\tau} \approx 0.02639, & A_{1}=A_{0} .
\end{array}\right.
$$

These values are quite close to the analytic one $c_{h}^{\text {an }}$ $\approx 0.02297$. The relative difference is $\left(c_{h}-c_{h}^{\mathrm{an}}\right) / c_{h}^{a n} \approx 0.164$ $\left(A_{1}=0\right)$ and $\left(c_{h}-c_{h}^{\mathrm{an}}\right) / c_{h}^{\mathrm{an}} \approx 0.149\left(A_{1}=A_{0}\right)$. Note that using the sum rules we overestimate the value of $c_{h}$. We can then expect to have overestimated the value of $c_{d}$, too. This remark will be important when comparing to numerical data.

Finally, we can evaluate condition (13) at late times, $t$ $\gg A_{0} / \lambda_{h}$, using the conjecture (8) for $n_{d}$ and the result $n_{h}(A, t) \sim c_{h} /\left(A+\lambda_{h} t\right)^{2}$ deduced from scaling in Sec. III A and to be shown analytically in Sec. IV A. In this case we find, independently of $A_{1}$,

$$
\frac{\lambda_{d}}{\lambda_{h}}=\frac{c_{d}}{c_{h}} \frac{1}{(\tau-1)} .
$$

This equation can be used to relate the two factors $\lambda_{d}$ and $\lambda_{h}$. Indeed, replacing $c_{d}$ and $c_{h}$ by their expressions as functions of $\tau$ one finds

$$
\frac{\lambda_{d}}{\lambda_{h}}= \begin{cases}1, & A_{1}=0, \\ 2^{\tau-2} \approx 1.019, & A_{1}=A_{0} .\end{cases}
$$

If, instead, we use $c_{d}$ as derived above and the analytic $c_{h}$, $c_{h}^{\text {an }}=0.022972$ we find

$$
1.164 \leqslant \frac{\lambda_{d}}{\lambda_{h}} \leqslant 1.170
$$

Since we derived Eq. (19) using the sum rules, it seems more appropriate to use the values of $c_{d}$ and $c_{h}$ obtained from the same relations. The values of $\lambda_{d}$ and $\lambda_{h}$ are then very close and consistent with the relation that we shall find in the next section using the approximate equation for the evolution of domain areas [while Eq. (21) yields a too large value for $\lambda_{d}$ ].

The condition $N_{p_{h}}(t)=N_{p_{d}}(t)=N_{h}(t)=N_{d}(t)$ implies

$$
\begin{gathered}
c_{p_{h}}=-c_{h}(1-\zeta), \\
c_{p_{d}}=c_{d} \frac{1-\zeta}{1-\tau},
\end{gathered}
$$

for critical Ising initial conditions.

\section{Infinite-temperature initial conditions}

The infinite-temperature initial conditions turn out to be, after just a few time steps, equivalent to critical percolation ones-see the numerical evidence in Sec. V A. The sum rules yield, in this case,

$$
\begin{gathered}
c_{d}=\frac{\left(\tau^{\prime}-2\right)\left(\tau^{\prime}-1\right)}{2} \approx 0.029, \\
c_{h}=\frac{c_{d}}{\left(\tau^{\prime}-1\right)} \approx 0.027,
\end{gathered}
$$

$$
\frac{\lambda_{d}}{\lambda_{h}}=\frac{c_{d}}{c_{h}\left(\tau^{\prime}-1\right)}=1,
$$

where, for simplicity, we present results obtained with $A_{1}$ $=0$ only.

The conditions $N_{p_{h}}(t)=N_{p_{d}}(t)=N_{h}(t)=N_{d}(t)$ imply

$$
c_{p_{h}}^{\prime}=-2 c_{h}\left(1-\zeta_{h}^{\prime}\right), \quad c_{p_{d}}^{\prime}=2 c_{d} \frac{1-\zeta_{d}^{\prime}}{1-\tau^{\prime}},
$$

for critical percolation.

\section{STATISTICS OF AREAS: ANALYTIC RESULTS}

Our analytic results are obtained using a continuum description of domain growth in which the nonconserved order parameter is a scalar field, $\phi(\vec{x}, t)$, defined on a $d$-dimensional space. For a review in this problem, see [1]. Its evolution is determined by the time-dependent GinzburgLandau equation or model $A$ dynamics,

$$
\gamma \frac{\partial \phi(\vec{x}, t)}{\partial t}=\nabla^{2} \phi(\vec{x}, t)-\frac{\delta V(\phi)}{\delta \phi(\vec{x}, t)}+\xi(\vec{x}, t) .
$$

The potential $V$ is a symmetric double well, with $V(\phi \rightarrow \pm \infty)=\infty$ and two minima at $\pm \phi_{0}$. $\xi$ is a Gaussian distributed random scalar field with zero mean and correlation

$$
\left\langle\xi(\vec{x}, t) \xi\left(\vec{x}^{\prime}, t^{\prime}\right)\right\rangle=2 k_{\mathrm{B}} T \gamma \delta^{d}\left(\vec{x}-\vec{x}^{\prime}\right) \delta\left(t-t^{\prime}\right) .
$$

This white noise introduces thermal agitation. $T$ is the temperature of the thermal bath, $k_{\mathrm{B}}$ is the Boltzmann constant, and $\gamma$ is the friction coefficient. From now on we set the units in such a way that $k_{\mathrm{B}}=\gamma=1$. The low-temperature ordering dynamics from a disordered initial condition corresponds to the growth of ordered domains of the two equilibrium states, $\phi(\vec{x}, t)= \pm \phi_{0}$, separated by interfaces. Using the evolution equation (27) at zero temperature, Allen and Cahn showed that in any dimension $d$ the velocity, $v$, of each element of a domain boundary is proportional to the local interfacial mean curvature, $\kappa[1,19]$,

$$
v=-\frac{\lambda_{h}}{2 \pi} \kappa
$$

$\lambda_{h}$ is a material constant with the dimensions of a diffusion constant, and the factor $1 / 2 \pi$ is for later convenience. The velocity is normal to the interface and points in the direction of reducing the curvature. The dynamics is then purely curvature driven at zero temperature.

Temperature fluctuations have a twofold effect. On the one hand they generate equilibrium thermal domains that are not related to the coarsening process. On the other hand they roughen the domain walls thus opposing the curvature driven growth and slowing it down. Then Eq. (29) is no longer valid. However, it has been conjectured and verified numerically that, at least for averaged dynamic quantities well described by the scaling hypothesis, all temperature effects are captured by introducing a $T$ dependent $\lambda_{h}$ parameter. We shall use this working hypothesis in the analytic part of our paper and we shall put it to the test numerically. 
The number density of hull enclosed or domain areas at time $t$ as a function of their initial distribution is

$$
n(A, t)=\int_{0}^{\infty} d A_{i} \delta\left[A-A\left(t, A_{i}\right)\right] n\left(A_{i}, t_{i}\right)
$$

with $A_{i}$ the initial area and $n\left(A_{i}, t_{i}\right)$ their number distribution at the initial time $t_{i}$. $A\left(t, A_{i}\right)$ is the hull enclosed/domain area, at time $t$, having started from an area $A_{i}$ at time $t_{i}$.

\section{A. Hull enclosed areas}

In two dimensions [20] we can immediately deduce the time dependence of the area contained within any finite hull by integrating the velocity around the hull,

$$
\frac{d A}{d t}=\oint v d l=-\frac{\lambda_{h}}{2 \pi} \oint \kappa d l=-\lambda_{h} .
$$

In the second equality we used the zero-temperature AllenCahn equation (29), and in the final one we used the GaussBonnet theorem. Integrating over time, with initial time $t_{i}$, we find

$$
A_{h}\left(t, A_{i}\right)=A_{i}-\lambda_{h}\left(t-t_{i}\right)
$$

Therefore

$$
\begin{aligned}
n_{h}(A, t) & =\int_{0}^{\infty} d A_{i} \delta\left[A-A_{i}+\lambda_{h}\left(t-t_{i}\right)\right] n_{h}\left(A_{i}, t_{i}\right) \\
& =n_{h}\left[A+\lambda_{h}\left(t-t_{i}\right), t_{i}\right] .
\end{aligned}
$$

In deriving this result we have implicitly assumed that a single domain cannot split into two, and that two domains cannot coalesce. A little thought shows that neither process is possible for two-dimensional curvature-driven growth since both processes require that two parts of a single domain boundary (for splitting) or parts of two different domain boundaries (for coalescence) come together and touch. But it is clear that the curvature-driven dynamics always acts to prevent this happening, since the velocities of the domain boundaries at the incipient contact point are in opposite directions.

The initial distributions, $n_{h}\left(A_{i}, t_{i}\right)$, are given by the CardyZiff results displayed in Eq. (1) — assuming, in the case of a quench from infinite temperature, that the system rapidly sets into the critical percolation condition (see Sec. V A). For $t$ $\gg t_{i}$ one immediately recovers the results in [5],

$$
\begin{aligned}
& n_{h}(A, t)=\frac{2 c_{h}}{\left(A+\lambda_{h} t\right)^{2}}, \quad T_{0} \rightarrow \infty, \\
& n_{h}(A, t)=\frac{c_{h}}{\left(A+\lambda_{h} t\right)^{2}}, \quad T_{0}=T_{c},
\end{aligned}
$$

in the limit $A_{0} \ll A \ll L^{2}$, i.e., for hull enclosed areas much larger than microscopic areas but much smaller than the area of the system.

Equations (34) and (35) have the expected scaling forms $n_{h}(A, t)=t^{-2} f(A / t)$ corresponding to a system with characteristic area proportional to $t$ or characteristic length scale
$R(t) \propto t^{1 / 2}$, which is the known result if scaling is assumed [1]. Here, however, we do not assume scaling-rather, it emerges from the calculation. Furthermore, the conventional scaling phenomenology is restricted to the "scaling limit" $A \rightarrow \infty, t \rightarrow \infty$ with $A / t$ fixed. Equations (34) and (35), by contrast, are valid whenever $t$ is sufficiently large and $A$ $\gg A_{0}$. This follows from the fact that, for large $t$, the forms (34) and (35) probe, for any $A \gg A_{0}$, the tail (i.e., the large- $A$ regime) of the Cardy-Ziff results, which is just the regime in which the latter is valid. The restriction $A \gg A_{0}$ is needed to justify the use of Eq. (29), which breaks down when the reciprocal of the curvature becomes comparable with the width of a domain wall.

The averaged area enclosed by a hull is then given by

$$
\begin{aligned}
\langle A\rangle(t) & =\frac{\int d A^{\prime} A^{\prime} n_{h}\left(A^{\prime}, t\right)}{\int d A^{\prime} n_{h}\left(A^{\prime}, t\right)} \\
& \propto \lambda_{h} t
\end{aligned}
$$

with a time-independent prefactor that behaves as $\left(A_{0}^{2} \ln L^{2}\right)$ for large system sizes. The reason for the divergent prefactor in the infinite size limit is that a site can belong to several hulls.

\section{B. Domains}

For the domains we need to write an evolution equation and derive, at least approximately, the area at time $t$, $A_{d}\left(t, A_{i}\right)$, of a domain with initial area $A_{i}$. We shall show that the time-dependent number density of domain areas is indeed given by our guess, Eqs. (8) and (9), for the two classes of initial conditions.

Our strategy is to exploit the smallness of the parameter $c_{h} \approx 0.023$. Although $c_{h}$ is a constant, we can exploit a formal expansion in $c_{h}$ in the following sense. Since the total number of hulls per unit area is proportional to $c_{h}$, the number of interior hulls within a given hull is also proportional to $c_{h}$, and so on. This means that, in dealing with domains we need consider only the first generation of interior hulls, since the number of "hulls within hulls" is smaller by a factor $c_{h}$. With this approach, only one approximation - a kind of mean-field one on the number of first-generation hulls within a parent hull (see below) - is necessary.

The same line of reasoning shows that, in a hypothetical theory in which $c_{h}$ can be treated as variable, the distinction between hulls and domains will disappear in the limit $c_{h}$ $\rightarrow 0$. In this limit, therefore, the exponents $\tau$ and $\tau^{\prime}$ must both approach the value 2 , i.e., we can formally write $\tau=2$ $+O\left(c_{h}\right)$ and $\tau^{\prime}=2+O\left(c_{h}\right)$. Furthermore, due to the factor 2 that appears in Eq. (34) but not in Eq. (35), the ratio $\left(\tau^{\prime}-2\right) /(\tau-2)$ must approach the value 2 in the limit $c_{h}$ $\rightarrow 0$. The actual value of this ratio is $187 / 91=\tau^{\prime}=2.055$, not very far from 2 . Indeed the difference is of order $c_{h}$ as expected.

We can use the same line of argument to discuss $c_{d}, c_{h}$, $\lambda_{d}$, and $\lambda_{h}$. Since in the (hypothetical) limit $c_{h} \rightarrow 0$, hulls and 


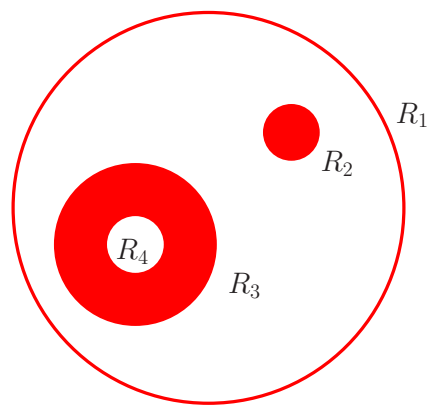

FIG. 3. (Color online) Sketch of a configuration with four circular hulls and domains. The parent hull has radius $R_{1}$. There are two first-generation hulls with radius $R_{2}$ and $R_{3}$ and one secondgeneration hull with radius $R_{4} . \nu=2$ in this example. The interior border of the external domain is disconnected and has two components.

domains become identical, it follows that in this limit one must have $c_{d} \rightarrow c_{h}$ and $\lambda_{d} \rightarrow \lambda_{h}$, i.e., $c_{d}=c_{h}+O\left(c_{h}^{2}\right)$, and $\lambda_{d}$ $=\lambda_{h}\left[1+O\left(c_{h}\right)\right]$. All of these results are consistent with the relations (15) and (19) derived from the sum rules (12) and (13).

\section{The evolution of domain areas}

Take a hull with enclosed area $A_{h}$ at time $t$. This hull is also the external border of a domain, which may itself contain one or more "first level" subdomains whose external borders form the internal border (which may be disconnected) of the original domain. These external borders of the first level subdomains are themselves "first-generation" hulls lying within the parent hull. These interior hulls can themselves have interfaces in their bulk separating domains of the reversed phase (higher generation hulls), see Fig. 3 where we show a sketch with this structure.

Let us call $\nu(t)$ the number of first-generation hulls within the parent one. It is clear that $\nu(t)$ is semipositive definite, monotonically decreasing as a function of time and reaching zero at a given instant $t_{\max }$, when all interior hulls disappear and $A_{d}=A_{h}$ thereafter. One can estimate $t_{\max }$ from 0 $=A_{h}^{\text {int }}\left(t_{\max }\right)=A_{h}^{\text {int }}\left(t_{i}\right)-\lambda_{h}\left(t_{\max }-t_{i}\right), \quad$ which yields $t_{\max }-t_{i}$ $=A_{h}^{\text {int }}\left(t_{i}\right) / \lambda_{h}$ where the index int indicates that we are studying here the first generation hull with maximal initial area (all others having already disappeared). It is clear that $t_{\max }$ $-t_{i}$ is smaller but of the order of $A_{h}\left(t_{i}\right) / \lambda_{h}$, where we replaced $A_{h}^{i n t}\left(t_{i}\right)$ by the initial area of the parent hull,

$$
\left(t_{\max }-t_{i}\right) \lessgtr \frac{A_{h}\left(t_{i}\right)}{\lambda_{h}} .
$$

We wish to write a differential equation for the time evolution of the parent domain area. It is clear that, at first order in $d t$,

$$
A_{d}(t+d t)=A_{d}(t)-\lambda_{h} d t+\nu(t) \lambda_{h} d t,
$$

where the second term in the right-hand side represents the loss in area due to the inward motion of the external domain wall while the last term is the gain in area due to the outward motion of the first-generation internal domain walls. This gives

$$
\frac{d A_{d}(t)}{d t}=-\lambda_{h}[1-\nu(t)]
$$

Differently from hull enclosed areas that always decrease in size as time passes, domain can either diminish $(\nu=0)$, increase $(\nu>1)$, or conserve $(\nu=1)$ their area in time.

\section{The number of first-generation interior hulls}

We cannot, of course, know the exact number of firstgeneration hulls falling within a selected hull with enclosed area $A_{h}$. We can, however, estimate it with an upper bound obtained by counting all interior hulls and averaging over all parent hulls using $n_{h}(A, t)$ derived in Sec. IV A. Thus, we expect

$$
\begin{gathered}
\nu(t)<\langle\nu(t)\rangle_{A_{h}(t)}, \\
\langle\nu(t)\rangle_{A_{h}(t)} \sim A_{h}(t) \int_{0}^{A_{h}(t)} d A n_{h}(A, t) \\
=\frac{c_{h} A_{h}^{2}(t)\left[\lambda_{h}\left(t-t_{i}\right)+A_{0}\right]^{-1}}{\left[A_{h}(t)+\lambda_{h}\left(t-t_{i}\right)+A_{0}\right]},
\end{gathered}
$$

where we include a small area cutoff, $A_{0}$, in the denominator of $n_{h}$ and, for concreteness, we use the hull enclosed area distribution for critical Ising initial conditions. This equation can be further simplified if one uses that at time $t$ the hull enclosed area we are interested in is given by

$$
A_{h}(t)=A_{h}\left(t_{i}\right)-\lambda_{h}\left(t-t_{i}\right) .
$$

[We call here $A_{h}\left(t_{i}\right)$ the initial area of the hull.] Then

$$
\langle\nu(t)\rangle_{A_{h}(t)}=\frac{c_{h}\left[A_{h}\left(t_{i}\right)-\lambda_{h}\left(t-t_{i}\right)\right]^{2}}{\left[\lambda_{h}\left(t-t_{i}\right)+A_{0}\right]\left[A_{h}\left(t_{i}\right)+A_{0}\right]} .
$$

Note that, although we overcounted the interior hulls by including second-generation, third-generation, etc. hulls, the number of these is of order $c_{h}^{2}, c_{h}^{3}, \ldots$, respectively, so this treatment is exact to leading order in $c_{h}$ except for the replacement of $\nu(t)$ by its average over all first-generation hulls of the same area.

The most interesting cases are such that $A_{h}\left(t_{i}\right) \gg A_{0}$, otherwise the hull and domain areas are just identical or very similar. In these cases $\left\langle\nu\left(t_{i}\right)\right\rangle_{A_{h}\left(t_{i}\right)} \sim c_{h} A_{h}\left(t_{i}\right) / A_{0}$. Expression (43) has the following limiting values:

$$
\langle\nu(t)\rangle_{A_{h}(t)} \sim \begin{cases}\frac{c_{h} A_{h}\left(t_{i}\right)}{\lambda_{h}\left(t-t_{i}\right)+A_{0}}, & A_{h}\left(t_{i}\right) \gg \lambda_{h}\left(t-t_{i}\right), \\ a c_{h}, & A_{h}\left(t_{i}\right) \sim \lambda_{h}\left(t-t_{i}\right) .\end{cases}
$$

We used $A_{h}\left(t_{i}\right) \gg A_{0}$ in the last case, and $a$ is a numerical constant of the order of $A_{h}\left(t_{i}\right)$. The result is a very small quantity, of the order of $c_{h}$, in both cases. The remaining mathematical possibility, $A_{h}\left(t_{i}\right)<\lambda_{h}\left(t-t_{i}\right)$, is not realized because $A_{h}(t)$ cannot be negative.

While $\nu(t)$ vanishes at $t_{\max }$, see Eq. (38), $\langle\nu(t)\rangle_{A_{h}(t)}$ is different from zero at all times. Thus, Eq. (43) cannot be used 


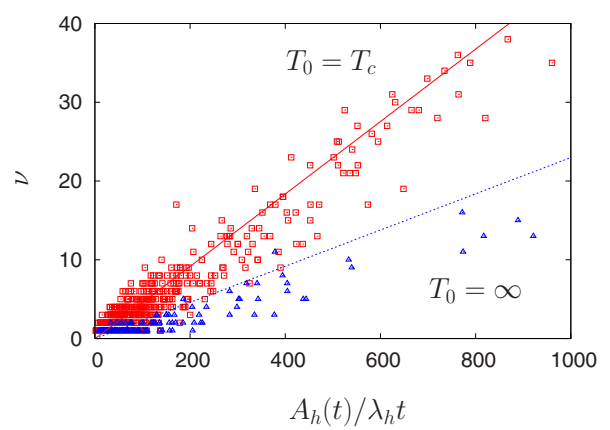

FIG. 4. (Color online) Comparison between $\nu(t)$ and $\langle\nu(t)\rangle_{A_{h}(t)}$ for the $T=0$ evolution of the $2 \mathrm{dIM}$ with $T_{0} \rightarrow \infty$ and $T_{0}=T_{c}$ initial conditions. The measuring time is $t=64 \mathrm{MCs}$. The curves are given by Eq. (41) in the limit $t \gg t_{i}$ and $A_{h}(t) \gg A_{0}$, leading to the functional form $\langle\nu(t)\rangle_{A_{h}(t)}=c_{h} x^{2} /(1+x) \sim c_{h} x$ when $x \gg 1$, with $x$ $=A_{h}(t) / \lambda_{h} t$ and $\lambda_{h}=2.1$, see Sec. V for an explanation of the choice of this value.

beyond the limit $t_{\max }$ when all internal hulls have already disappeared and it is no longer correct to replace $\nu(t)$ by $\langle\nu(t)\rangle_{A_{h}(t)}$.

The analysis of infinite temperature initial conditions is identical to the one above with $c_{h}$ replaced by $2 c_{h}$. Thus, $\langle\nu(t)\rangle_{A_{h}(t)}$ is expected to take twice the value it takes for critical Ising initial configurations.

We have checked the accuracy of this approximation numerically by counting the number of first generation internal hulls falling within each parent hull at different times. Figure 4 shows the results for the zero-temperature evolution of the $2 \mathrm{dIM}$ starting from $T_{0} \rightarrow \infty$ and $T_{0}=T_{c}$ initial conditions. While at very short times one sees deviations between the numerical data and analytic prediction, the agreement between the two becomes very satisfactory for times of the order of $t=64 \mathrm{MCs}$ and longer, as shown in the figure.

\section{The instantaneous domain area}

If we now replace $\nu(t)$ by $\langle\nu(t)\rangle_{A_{h}(t)}$ given in Eq. (43), it is quite simple to integrate the differential equation (40). One finds

$$
\begin{aligned}
A_{d}(t)= & A_{d}\left(t_{i}\right)-\lambda_{h}\left(1+2 c_{h}\right)\left(t-t_{i}\right)+\frac{c_{h} A_{0}^{2}}{2\left[A_{h}\left(t_{i}\right)+A_{0}\right]} \\
& \times\left\{\left[1+\frac{\lambda_{h}\left(t-t_{i}\right)}{A_{0}}\right]^{2}-1\right\}+c_{h}\left[A_{h}\left(t_{i}\right)+A_{0}\right] \ln \\
& \times\left[1+\frac{\lambda_{h}\left(t-t_{i}\right)}{A_{0}}\right]
\end{aligned}
$$

Setting $t=t_{i}$ one recovers $A_{d}(t)=A_{d}\left(t_{i}\right)$ as required. In the natural cases in which $A_{h}\left(t_{i}\right) \gg A_{0}$ and for long times such that $\lambda_{h}\left(t-t_{i}\right) \gg A_{0}$, this expression can be rewritten as

$$
\begin{aligned}
A_{d}(t)= & A_{d}\left(t_{i}\right)-\lambda_{h}\left[1+2 c_{h}-\frac{c_{h}}{2} \frac{\lambda_{h}\left(t-t_{i}\right)}{A_{h}\left(t_{i}\right)}\right]\left(t-t_{i}\right) \\
& +c_{h} A_{h}\left(t_{i}\right) \ln \left[1+\frac{\lambda_{h}\left(t-t_{i}\right)}{A_{0}}\right] .
\end{aligned}
$$

The factor in the second term

$$
\lambda_{d}(t) \equiv \lambda_{h}\left[1+2 c_{h}-\frac{c_{h}}{2} \frac{\lambda_{h}\left(t-t_{i}\right)}{A_{h}\left(t_{i}\right)}\right]
$$

is a very weakly time-dependent function. Since $t$ can take values between the initial time, $t=t_{i}$, and the maximum time before the first-generation hull itself disappears, $t_{\max }=t_{i}$ $+A_{h}\left(t_{i}\right) / \lambda_{h}, \lambda_{d}(t)$ varies within the interval,

$$
\lambda_{h}\left(1+\frac{3 c_{h}}{2}\right) \leqslant \lambda_{d}(t) \leqslant \lambda_{h}\left(1+2 c_{h}\right)
$$

These bounds are indeed very close. As expected from the analysis of the sum rules, see Sec. III, $\lambda_{d}$ takes a slightly higher value than $\lambda_{h}$; it equals $\lambda_{h}$ plus a small correction of order $c_{h}$ (in practice, $1.035 \lambda_{h} \leqslant \lambda_{d} \leqslant 1.046 \lambda_{h}$ using the analytic value for $c_{h}$ ).

The coefficient in front of the logarithm, $c_{h} A_{h}\left(t_{i}\right)$, is $\mathcal{O}\left(c_{h}\right)$. The sum rules, imply $c_{h}=c_{d}+\mathcal{O}\left(c_{h}^{2}\right)$. Neglecting the higher-order correction we can then replace $c_{h}$ by $c_{d}$. The same applies to $A_{h}\left(t_{i}\right)$, which equals $A_{d}\left(t_{i}\right)$ plus a term $\mathcal{O}\left(c_{h}\right)$ that we can equally neglect. Thus $A_{h}\left(t_{i}\right) \approx A_{d}\left(t_{i}\right) \equiv A_{i}$. In this way we obtain

$$
A_{d}\left(t, A_{i}\right) \simeq A_{i}-\lambda_{d}\left(t-t_{i}\right)+c_{d} A_{i} \ln \left[1+\frac{\lambda_{h}\left(t-t_{i}\right)}{A_{0}}\right] .
$$

Inserting this result into Eq. (30), including the microscopic area $A_{0}$ as a small-area cutoff in the denominator of Eq. (1), then gives

$$
\begin{aligned}
n_{d}(A, t) \simeq & c_{d} A_{0}^{\tau-2}\left\{1+c_{d} \ln \left[1+\frac{\lambda_{d}\left(t-t_{i}\right)}{A_{0}}\right]\right\}^{\tau-1} \\
& \times\left[A+\lambda_{d}\left(t-t_{i}\right)+A_{0}\right]^{-\tau},
\end{aligned}
$$

where we have replaced $\lambda_{h}$ by $\lambda_{d}$ inside the logarithm, which is correct to leading order in $c_{h}$. Using the fact that $c_{d}$ is very small and of the order of $(\tau-2)(\tau-1)=(\tau-2)+\mathcal{O}\left(c_{h}^{2}\right)$, as implied by the sum rules, we can now exponentiate, correct to leading order in $c_{d}$, the logarithm in the curly brackets to obtain

$$
n_{d}(A, t) \simeq \frac{c_{d}\left[A_{0}+\lambda_{d}\left(t-t_{i}\right)\right]^{\tau-2}}{\left[A+A_{0}+\lambda_{d}\left(t-t_{i}\right)\right]^{\tau}} .
$$

Finally we set the initial time, $t_{i}$, to zero and write the microscopic area, $A_{0}$, as $\lambda_{d} t_{0}$ to obtain the expected form (8),

$$
n_{d}(A, t) \simeq \frac{c_{d}\left[\lambda_{d}\left(t+t_{0}\right)\right]^{\tau-2}}{\left[A+\lambda_{d}\left(t+t_{0}\right)\right]^{\tau}},
$$

for the time-dependent number density of domain areas.

The same sequence of steps for infinite-temperature initial conditions leads to the same form but with $c_{d}$ replaced by $2 c_{d}$ and $\tau$ replaced by $\tau^{\prime}$. The effects of temperature are expected to appear only through the parameters $\lambda_{d}$ and $\lambda_{h}$ once thermal fluctuations are extracted from the analysis.

The averaged domain area is then given by

$$
\langle A\rangle(t)=\frac{\int d A^{\prime} A^{\prime} n_{d}\left(A^{\prime}, t\right)}{\int d A^{\prime} n_{d}\left(A^{\prime}, t\right)}=\frac{1}{N_{d}(t)}
$$


$\propto \lambda_{d} t$.

\section{STATISTICS OF AREAS: NUMERICAL TESTS}

To test our analytic results we carried out numerical simulations on the 2D square-lattice Ising model (2dIM) with periodic boundary conditions using a heat-bath algorithm with random sequential updates. All data have been obtained using systems with size $L^{2}=10^{3} \times 10^{3}$ and $2 \times 10^{3}$ runs using independent initial conditions.

Domain areas are identified with the Hoshen-Kopelman algorithm [21] while hull-enclosed ones are measured by performing a directed walk along the interfaces, in analogy with the algorithm in [15]. A detailed description of our algorithm is given in Appendix A.

The equilibrium critical Ising initial conditions have one spanning cluster (since the system is at the limit of the percolation threshold), that grows during evolution. No other spanning cluster is later formed. By contrast, equilibrium infinite temperature initial conditions are below the critical random percolation point in $d=2$ but often after $2 \mathrm{MC}$ steps two spanning clusters appear that also grow during evolution. After $20 \mathrm{MCs}$ roughly $50 \%$ of the spins lie typically on these clusters. This implies that we need to simulate a large number of independent samples to obtain a good statistics.

It is important to note that the dynamics of the discrete model includes processes that are not taken into account in the continuous model, as given in Eq. (29), for which we derived our analytical results. Some of these processes are the fission of a big domain into two smaller ones (that usually occurs by cutting a thin bottle neck that joined them), or the coalescence of two domains to form a bigger one. However, we shall prove that these processes are not important and the dynamics of the discrete model is well described by the analytic results.

\section{A. Initial conditions}

We used three types of initial conditions: equilibrium at infinite temperature, $T_{0} \rightarrow \infty$; equilibrium at the critical point, $T_{0}=T_{c}$; equilibrium within the high-temperature phase, $T_{0}$ $=T_{c}+\Delta T$ with $\Delta T>0$.

We mimicked an instantaneous quench from $T_{0} \rightarrow \infty$ by using random initial states with spins pointing up or down with probability $1 / 2$. Assigning site occupation to up spins and vacant sites to down spins the infinite temperature initial condition can be interpreted as a percolation problem at $p$ $=0.5$ and thus below the random percolation transition $p_{c}$ $=0.5927$ in a square bidimensional lattice. Even if initially away from criticality, in a few MC steps the hull enclosed area distribution becomes the one in Eq. (1), as shown in panel (a) of Fig. 5. The initial distribution lacks large areas, there being almost none with $A>10^{3}$, and the tail of $n_{h}$ falls off too quickly well below the critical percolation curve. In a few time steps large structures appear and the tail of the distribution approaches the expected form at critical percolation. Simultaneously, the weight at small areas diminishes and the curve progressively gets flatter. This effect can also be seen in Fig. 25 where we display data for perimeter

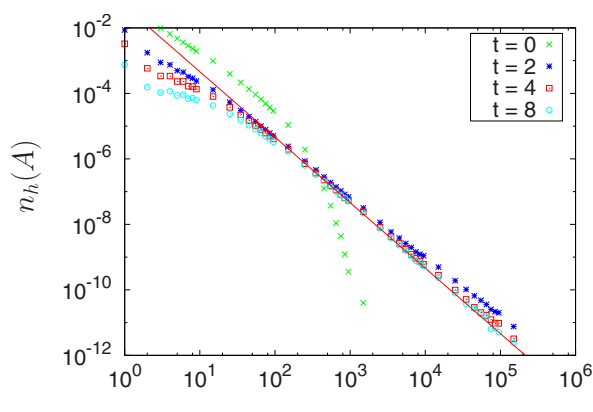

(a)

A

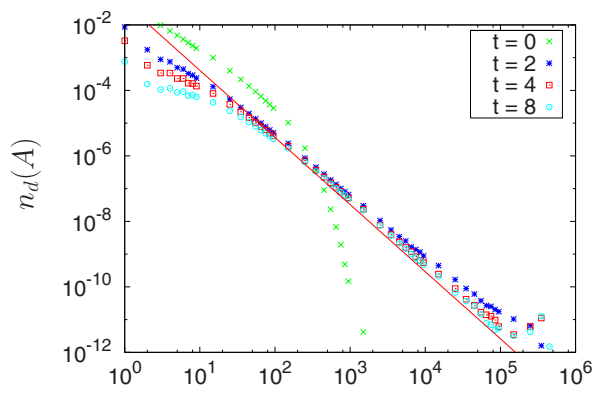

(b)

A

FIG. 5. (Color online) Early evolution of the infinite temperature initial condition. (a) In a few MC steps the hull enclosed area distribution reaches the one of critical percolation (1), whose slope, -2 , is shown by the straight line [6]. (b) The domain area distribution. The straight line is the power-law decay $A^{-2.055}[10]$.

lengths. In panel (b) of Fig. 5 we display our numerical results for the domain area distribution, which are compatible with the form (2). It is intuitively clear why this must be so. If the system is coarse grained on the domain typical scale, $R(t)$, it will look completely disordered. When $R(t)$ is large compared to the lattice spacing, the disorder will be that of continuum percolation, for which the critical density is one half by symmetry in two dimensions $[22,23]$. It follows that the coarsening system will be asymptotically at percolative criticality, i.e., the dynamics self-tunes the system to percolative criticality in two dimensions [provided $R(t)$ remains much smaller than the system size]. The data show that, as far as the hull and domain area distributions are concerned, this only takes a few Monte Carlo steps in practice. During these few steps many small domains coalesce to form larger ones meaning that the dynamics is dominated by processes that are not taken into account by Eq. (29). This argument also shows that the domain distribution in the scaling limit indeed has the predicted $A^{-\tau^{\prime}}$ tail. It is interesting to remark that the system approaches the percolative critical state not by increasing $p$ (indeed, the magnetization during the coarsening process initially remains close to zero), but by decreasing the value of $p_{c}$, from 0.5927 to 0.5 , as the correlation between spins increases.

We can look at this from another perspective in the context of the continuum model. Consider a random field $\phi(\vec{x})$, symetrically distributed with respect to zero, with bounded variance and two-point correlator $C(r)=\langle\phi(\vec{x}) \phi(\vec{x}+\vec{r})\rangle$ with $r=|\vec{r}|$. The zero contour lines of this field can be imagined to 


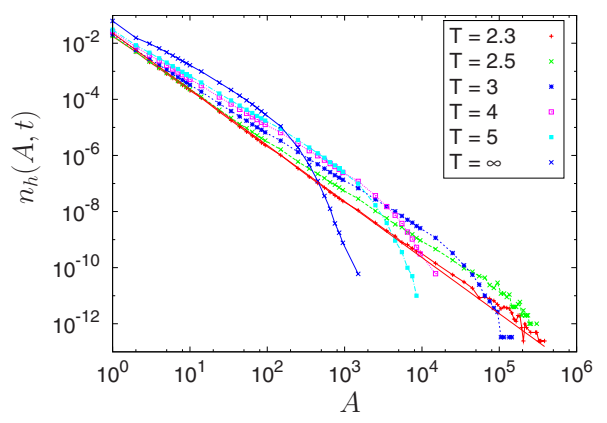

FIG. 6. (Color online) Equilibrium number density of hulls per unit area for the high-temperature phase of the 2dIM. The distributions seem power law for small areas, with a temperature-dependent exponent that approaches 2 when $T \rightarrow T_{c}$. Indeed, data for $T=2.3$ $\simeq 1.01 T_{c}$ is almost coincident with Eq. (35).

divide the plane into regions of black and white with each contour line forming a boundary between black and white regions. Provided that $C(r)$ falls off faster than $r^{-3 / 4}$ for large $r$, this problem is known to belong to the standard percolation universality class [23]. If we now identify $\phi(\vec{x})$ with the order parameter field when well-defined domain walls (the zero contour lines) have formed, we see that the resulting domain structure corresponds to critical percolation.

In the plots we use a double logarithmic scale that serves as a first check of the power-law decay of the probability distributions but it is not accurate enough to examine the value of the constants $c_{h}$ and $c_{d}$. We delay the presentation of a very precise test of these parameters to Sec. V B where we analyze the time evolution of the distribution functions.

We obtained the initial states for the coarsening dynamics at the critical temperature, $T_{0}=T_{c}$, and at $T_{0}=T_{c}+\Delta T$ (see Fig. 6) after running $10^{3}$ Swendsen-Wang algorithm steps. We checked that the systems are well equilibrated after these runs. The distribution of hull enclosed and domain areas at $T_{c}$ are consistent with the analytic forms-not shown.

\section{B. Coarsening at zero temperature}

\section{Hull enclosed areas}

In Fig. 7 we show the time-dependent hull enclosed area

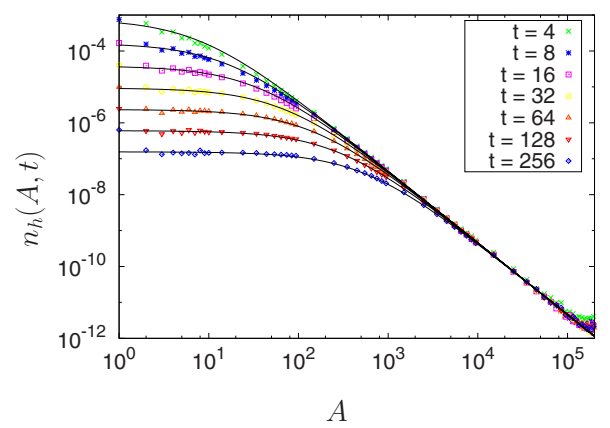

FIG. 7. (Color online) Number density of hull enclosed areas per unit system area for the zero-temperature dynamics of the 2dIM at seven times evolving from an infinite temperature initial condition. The lines represent Eq. (34) with $c_{h}=1 / 8 \pi \sqrt{3}$.

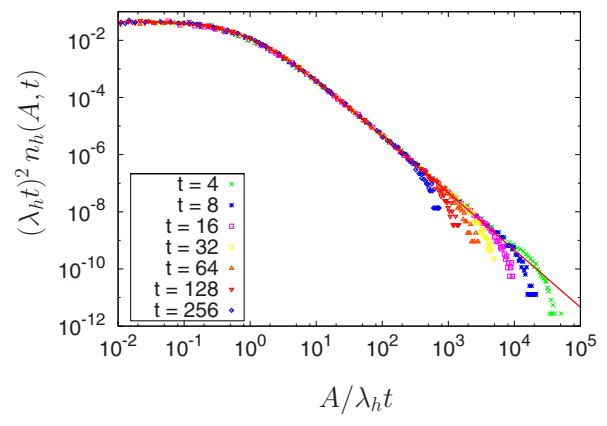

FIG. 8. (Color online) Number density of hull enclosed areas per unit system area for the zero-temperature dynamics of the 2dIM evolving from an infinite temperature initial condition. The full line is the prediction (34) with $c_{h}=1 / 8 \pi \sqrt{3}$ and $\lambda_{h}=2.1$.

distribution in double logarithmic scale, at seven different times, following a quench from $T_{0} \rightarrow \infty$. The figure shows a strong time dependence at small areas and a very weak one on the tail, which is clearly very close to a power law. The curves at small areas move downwards and the breaking point from the asymptotic power law decay moves towards larger values of $A$ for increasing $t$.

In Fig. 10 (b) we zoom on the small area region $(A$ $<10^{3}$ ) where the time dependence is clearer and we scale the data by plotting $\left(\lambda_{h} t\right)^{2} n_{h}(A, t)$ against $A / \lambda_{h} t$ with $\lambda_{h}=2.1$. We tried other time-dependent factors but $\lambda_{h} t$ with this particular value of $\lambda_{h}$ is the one yielding the best collapse of data at small areas, $A_{0} \ll A<\lambda_{h} t$. For $A$ larger than the typical value $\lambda_{h} t$ the time and $\lambda_{h}$ dependence becomes less and less important. In Fig. 8 we show the data in their full range of variation in log-log form to test the prediction $n_{h}(A, t) \propto A^{-2}$ for large $A$. The data are in remarkably good agreement with the prediction (34) - shown as a continuous curve in the figure-over the whole range of $A$ and $t$. The downward deviations from the scaling curve are due to finite-size effects. The latter are shown in more detail in Fig. 9, where we display the $t=16 \mathrm{MCs}$ results for several linear sizes. Finite size effects appear only when the weight of the distribution has fallen by many orders of magnitude ( 7 for a system with $L=10^{3}$ ) and are thus quite irrelevant. In the tail of the probability distribution function (pdf) the numerical error is

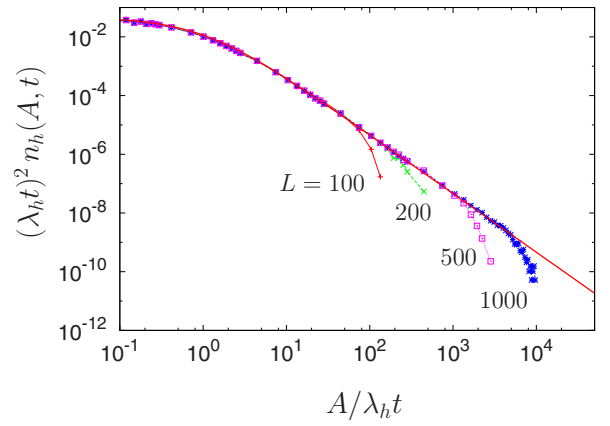

FIG. 9. (Color online) Finite size effects at $t=16 \mathrm{MCs}$; four linear sizes of the sample are used and indicated by the data points. The value of $A / t$ at which the data separate from the master curve grows very fast with $L$ with an exponent close to 2 . 

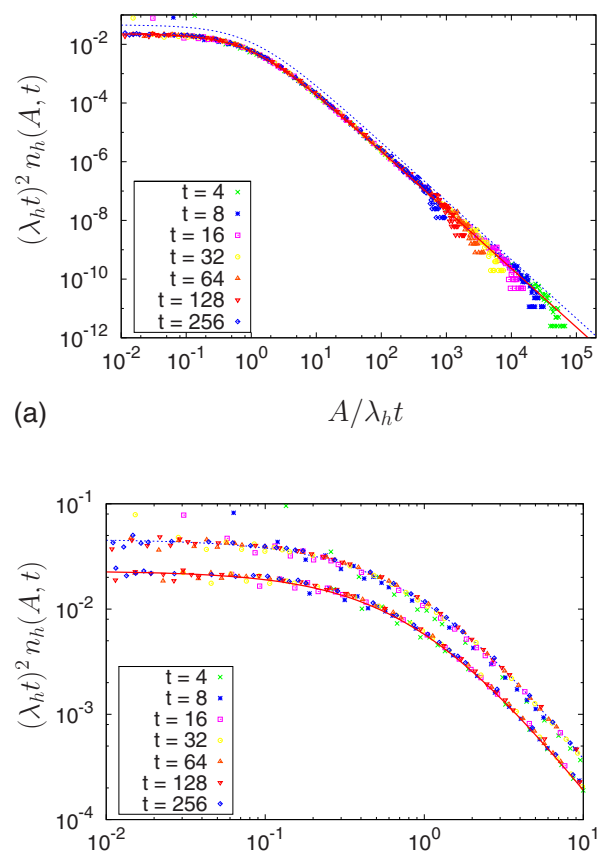

(b)

$A / \lambda_{h} t$

FIG. 10. (Color online) Number density of hulls per unit area for the zero-temperature 2dIM evolving from critical initial conditions. The initial states are obtained after running $10^{3}$ SwendsenWang algorithm steps. (a) The full (red) line is Eq. (35) with $\lambda_{h}$ $=2.1$ which again yields the best fit of the data at small areas. For comparison we include with a dotted (blue) line the analytic prediction for an infinite temperature initial condition, i.e., Eq. (34). (b) More details on the influence of the initial conditions. The two data sets correspond to configurations taken at several times after a quench from $T_{0} \rightarrow \infty$ and $T_{0}=T_{c}$. The solid lines are the analytic predictions (34), blue line, and (35), red line.

smaller than the size of the data points. The nearly perfect agreement between the analytical theory and the data is all the more impressive given that the curvature-driven growth underlying the prediction (34) only holds in a statistical sense for the lattice Ising model [24]. Indeed, even at small values of $A / \lambda_{h} t$, where the lattice and continuous descriptions are expected to differ most, the difference is only a few percent, as we shall show below.

It is clear that the evolution of the hull enclosed area distribution follows the same advection law (33), with the same value of $\lambda_{h}$, for other initial conditions. The evolution from a critical temperature initial condition is shown in panel (a) of Fig. 10. A fit of the data at small areas yields the value of the parameter $\lambda_{h}$ that, consistently with the analytic prediction, takes the same value $\lambda_{h}=2.1$. In the bottom panel, we compare the time-dependent hull enclosed area distributions for the initial conditions $T_{0} \rightarrow \infty$ and $T_{0}=T_{c}$ and we zoom on the behavior of $n_{h}(A, t)$ at small areas, $A / \lambda_{h} t \leqslant 10$. The two solid lines correspond to the numerator in $n_{h}$ being equal to $2 c_{h}$ for infinite temperature initial conditions and $c_{h}$ for critical Ising initial conditions. The difference between the numerical data for the two initial states is clear and it goes in the direction of the analytic prediction (a factor 2 difference in the constant). Finally, while the log-log plot in

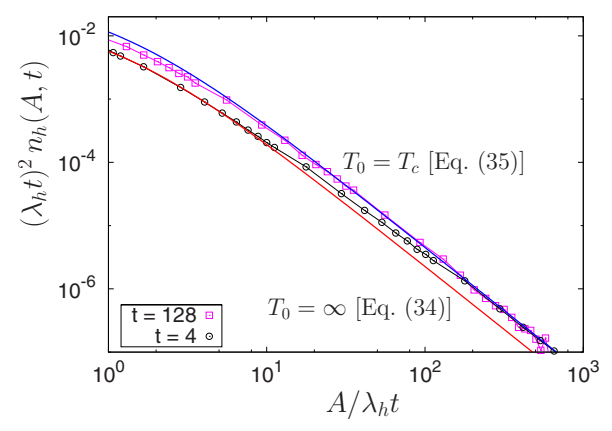

FIG. 11. (Color online) Effect of the initial condition temperature on the hull enclosed area distribution. The continuous lines are the analytic results for equilibrium initial conditions at $T_{0}=\infty$ and $T_{0}=T_{c}$, bottom (red) and top (blue) lines, respectively. In between, we present numerical data for two different times given in the key after the quench from an initial state equilibrated at $T_{0}=2.5$.

Fig. 8 suggests that the data are compatible with $c_{h} \approx 0.023$ this way of presenting the data is not precise enough to let us quantify the accuracy with which we match the analytic prediction. We test the numerical values of the constant $c_{h}$ in detail in Sec. V where the numerical error is also estimated.

Moreover, Eq. (34) applies to any $T_{0}>T_{c}$ equilibrium initial condition asymptotically. Equilibrium initial conditions at different $T_{0}>T_{c}$ show only a different transient behavior: the closer they are from $T_{c}$, the longer it takes to reach the asymptotic law, Eq. (34). Equilibrium initial distributions, for $T_{c}<T_{0}<\infty$, are shown in Fig. 6, while Fig. 11 shows an example of their subsequent evolution. In the latter, both analytic predictions, for $T_{0}=T_{c}$ and $T_{0}=\infty$, are shown as solid lines along with data for increasing times after a zerotemperature quench from $T_{0}=2.5$. In the first steps, the curve follows the one for critical initial conditions at small $A / \lambda_{h} t$ and then departs to reach the one for infinite temperature initial conditions at large $A / \lambda_{h} t$. At longer times, the deviation from the critical initial condition line occurs at a smaller value of $A / \lambda_{h} t$. Initially the system has a finite, though relatively small, correlation length $\xi(T)$. Thermal fluctuations with linear size of the order of $\xi$ and also significantly larger than $\xi$ exist (see the discussion on the effect of thermal fluctuations in Sec. V C). Notice that $\xi(T)$ does not correspond exactly to the size of geometric domains: thermal fluctuations are not perfectly described by domains of aligned spins, since not all of them are correlated. At any given temperature above $T_{c}$, fluctuations smaller than $\xi(T)$ have the same statistics than those occurring at $T_{c}$ and are thus described by Eq. (35), while domains larger than $\xi(T)$ are not made of correlated spins and thus are described by the infinite temperature distribution, Eq. (34). As time increases, the system loses memory of the finite-size fluctuations and the asymptotic state does not differ from the infinite temperature one. Only when fluctuations exist over all spatial scales does the asymptotic state differ.

This behavior can be interpreted as follows. At fixed $A / t$, shorter times correspond to small areas while longer times are related to larger areas. Very small areas correspond to short linear sizes, of the order of the domains in the initial configurations, and thus reminiscent of critical ones. Instead 

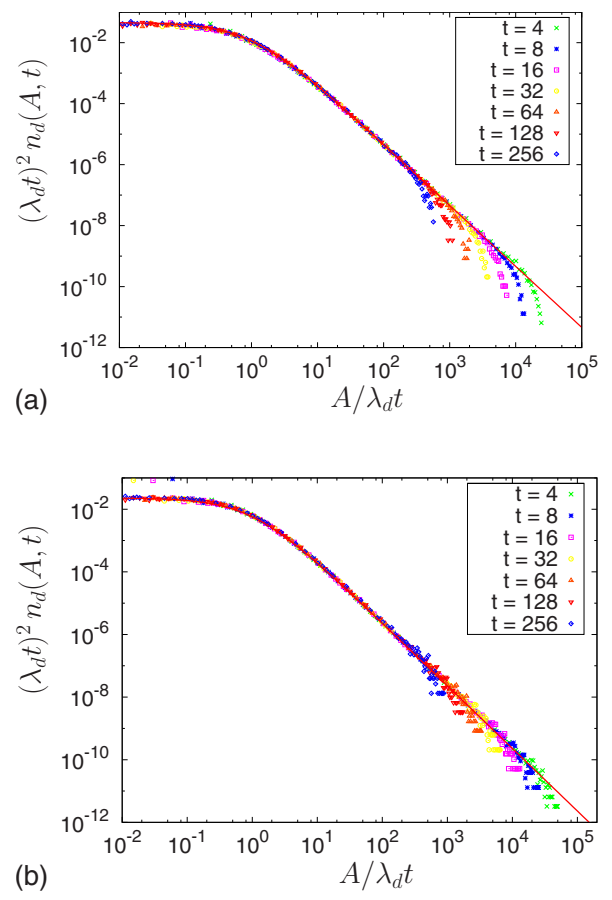

FIG. 12. (Color online) Number density of domains per unit area for the zero-temperature 2dIM evolving from $T_{0} \rightarrow \infty$ (a) and $T_{0}=T_{c}$ (b) initial conditions. In both figures the spanning clusters have been extracted from the analysis (compare with Fig. 13 where we include them). The full (red) line represents Eq. (49), with $c_{d}$ $=0.025$ and $\tau^{\prime}=2.055$ (a), $c_{d} \rightarrow c_{d} / 2$ and $\tau=2.027$ (b), and $\lambda_{d}=2.1$ in both cases.

large areas correspond to long linear sizes that are much longer than the correlation length and closer to the ones reached from the infinite temperature initial condition.

\section{Domain areas}

We now study the domain areas and perform the same tests as above though focusing on the analytic predictions (8) and (9).

In Fig. 12 we display the number density of domain areas in the scaled form for two initial conditions, $T_{0} \rightarrow \infty$ and $T_{0}$ $=T_{c}$, after removing any spanning domain from the statistics. For comparison, in Fig. 13, the same distributions with the spanning domains are shown. As done for the hull enclosed areas we fit the parameter $\lambda_{d}$ by analyzing the behavior at small areas, $A<\lambda_{d} t$, and we find, once again, that $\lambda_{d}=2.1$ yields the best collapse of data (see the discussion in Sec. IV B). We use the extrapolated value $c_{d}=0.025$ obtained with the numerical analysis described in full detail in Sec. V B 3. Note that we expect the difference between $c_{d}$ and $c_{h}$ to be of order $c_{h}^{2}$, and thus rather hard to observe numerically.

Both sets of figures, 12 and 13, exhibit finite size effects in the tail of the distributions, where the number of domain areas has already decreased by several orders of magnitude. As for the hull enclosed areas, the point where these finite size effects cause the deviation from the collapsed curve moves towards the right as the system size increases, becoming less and less relevant. In Fig. 12, large domain areas
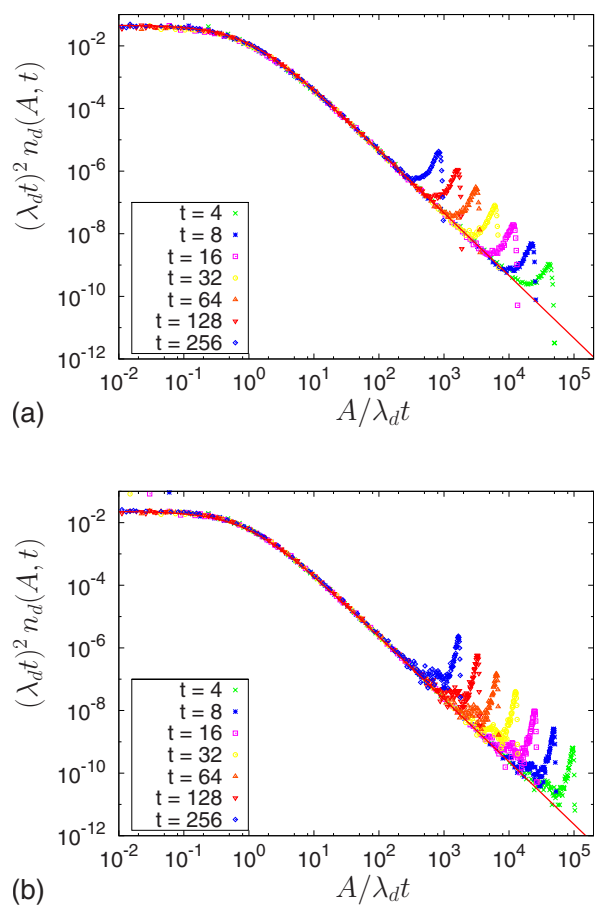

FIG. 13. (Color online) The same as Fig. 12, but with the spanning domains included in the statistics. Notice that due to the finiteness of the lattice, the actual area of these spanning domains are smaller than the value they would have on an infinite system, generating the overshoot of the distribution for values of $A$ close to $L^{2}$. The larger the system, the more to the right these peaks are localized.

(violating the limiting condition $A \ll L^{2}$ ) that would nonetheless be accounted for in an infinite system are here removed since they span the system in one of the directions, leading to the downward bending of the distribution. On the other hand, in Fig. 13, when counting these domains, they are chopped by the system boundaries, thus contributing to the distribution in a region shifted to the left, accounting for the bumps seen in the figure.

\section{Study of the constants $c_{h}$ and $c_{d}$}

In order to improve the data analysis we followed the procedure used by Cardy and Ziff, who studied the finite area scaling of the cumulative distribution between $A$ and $2 A$ [6]. The method is as follows. For hull enclosed and domain areas the total number of areas between $A$ and $2 A$ is $N_{h, d}(A, 2 A)=\int_{A}^{2 A} d A^{\prime} n_{h, d}\left(A^{\prime}\right)$. Using the analytic prediction for $n_{h}$ one finds $2 A N_{h}(A, 2 A) \sim c_{h}$ for large areas at critical Ising conditions and without the factor 2 at critical percolation. Following Ref. [6], we assume that there are power-law finite area corrections and add a term such as $a A^{-b}$ to the above expressions. From this relation one extracts the value of $c_{h}$. Similarly, for domains one can use $(1-\tau) N_{d}(A, 2 A) /\left[(2 A)^{1-\tau}-A^{1-\tau}\right]=c_{d}+a A^{-b}$ at critical Ising initial and its modified form at critical percolation initial conditions.

In the dynamic case we have 


$$
N_{h, d}(A, 2 A ; t) \equiv N_{h, d}(A, t)-N_{h, d}(2 A, t)=\int_{A}^{2 A} d A^{\prime} n_{h, d}\left(A^{\prime}, t\right) .
$$

Using the predictions for $n_{h, d}$ we find

$$
N_{h}(A, 2 A ; t)=\frac{2 c_{h} A}{\left(A+\lambda_{h} t\right)\left(2 A+\lambda_{h} t\right)}
$$

and

$$
N_{d}(A, 2 A ; t)=\frac{2 c_{d}\left(\lambda_{d} t\right)^{\tau^{\prime}-2}}{1-\tau^{\prime}}\left[\left(2 A+\lambda_{d} t\right)^{1-\tau^{\prime}}-\left(A+\lambda_{d} t\right)^{1-\tau^{\prime}}\right]
$$

for $T_{0} \rightarrow \infty$ and without the factor 2 and with the exponent $\tau^{\prime}$ replaced by $\tau$ for $T_{0}=T_{c}$. To extract the values of the constants $c_{h}$ and $c_{d}$, we rewrite these forms as

$$
(2 A)^{-1}\left(A+\lambda_{h} t\right)\left(2 A+\lambda_{h} t\right) N_{h}(A, 2 A ; t)=c_{h}+a\left(\frac{A}{\lambda_{h} t}\right)^{-b}
$$

and

$$
\frac{\left(\lambda_{d} t\right)^{2-\tau^{\prime}}\left(1-\tau^{\prime}\right) N_{d}(A, 2 A ; t)}{2\left[\left(2 A+\lambda_{d} t\right)^{1-\tau^{\prime}}-\left(A+\lambda_{d} t\right)^{1-\tau^{\prime}}\right]}=c_{d}+a\left(\frac{A}{\lambda_{d} t}\right)^{-b}
$$

and similarly for $T_{0}=T_{c}$. $a$ is a constant that takes different values for different times. In Figs. 14-16 we show the outcome of this analysis. We use the same scale on the vertical axis in all plots to compare the accuracy of the results.

In Fig. 14 we show results for the hull enclosed areas. In the top panel we study equilibrium data at $T_{c}$. The extrapolation of the numerical data approaches the analytic prediction for $c_{h}$ within a $2 \%$ accuracy. We use the value of the exponent $b$ proposed by Cardy and Ziff [6]. The estimation from the approximate use of the sum rules is well above the analytic prediction and numerical value. In the bottom panel we test the value of $c_{h}$ in our dynamic prediction by studying data at three instants, $t=16,64,128 \mathrm{MCs}$, after a critical Ising initial configuration. In this analysis we use the same exponent, $b=0.875$, as in the study of the equilibrium data and we fit the other parameters, $c_{h}$ and $a$. The fits of the time-dependent data to straight lines extrapolate to the same value that is, however, of the order of $5 \%$ off the analytic prediction. Note the nonmonotonic character of the timedependence in the slopes $a$. One has to keep in mind that the extrapolated value is very sensitive to the fit, in particular, to how many data points are considered.

In the analysis of the infinite temperature initial conditions we are forced to use dynamic results to reach, first the critical percolation situation, and next follow the coarsening evolution. In Fig. 15 we display this type of data for the instants given in the key. The prediction from the fit is slightly different from the analytic result at short times but it approaches the analytic value, shown with a horizontal line, at sufficiently long times (look at the $t=128 \mathrm{MCs}$ results). In conclusion we find
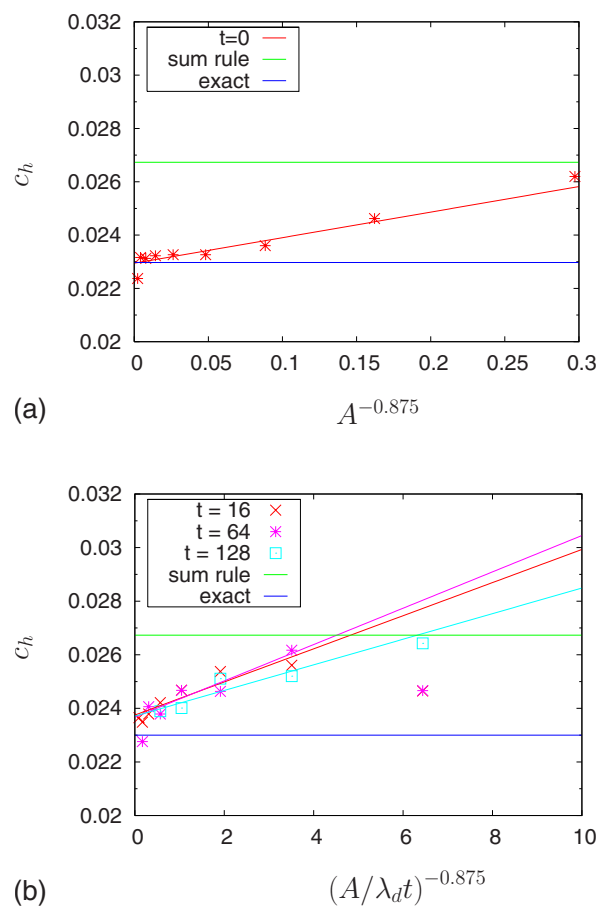

FIG. 14. (Color online) Study of $c_{h}$ using finite area scaling implemented as in [6]. The numerical data correspond to $2 A N_{h}(A, 2 A)$ in the equilibrium case and the left-hand side of Eq. (56) in the dynamic case. (a) Equilibrium distribution of hull enclosed areas at criticality. The upper horizontal line is the predictions for $c_{h}$ stemming from the use of the sum rule. The lower horizontal line is the analytic prediction. The intercept of the inclined straight line with $x=0$ is the numerical test of the analytic prediction. (b) Study of $c_{h}$ during coarsening after a quench from criticality to zero temperature. Note that the prediction from the fit is slightly higher than the analytic value, see the discussion in the text.

$$
c_{h} \sim 0.0229 \pm 0.0015 .
$$

Next we study the constant $c_{d}$. First we use critical temperature initial conditions using the dynamic results to avoid the ambiguity introduced by the units restoring constant $A_{0}$ in equilibrium. In short we find

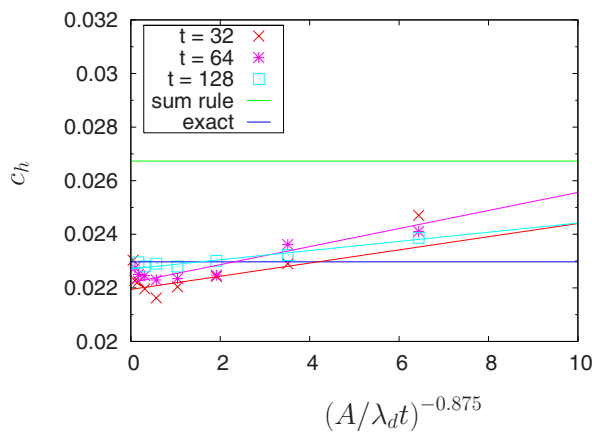

FIG. 15. (Color online) Study of $c_{h}$ during coarsening after a quench from $T_{0} \rightarrow \infty$ to $T=0$ using finite-area scaling implemented as in [6]. Note that the prediction from the fit weakly depends on time. 


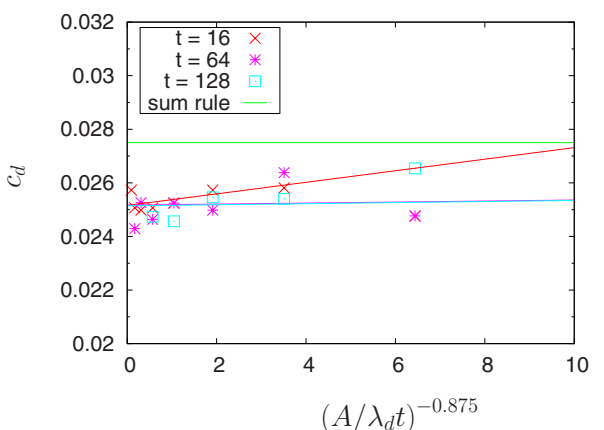

FIG. 16. (Color online) Study of $c_{d}$ using finite area scaling implemented as in [6]. $c_{d}$ extracted from time-dependent pdf evolving at $T=0$ after a quench from criticality. The numerical value of $c_{d}$ is roughly the same for all times.

$$
c_{d} \sim 0.0251 \pm 0.0015 \text {. }
$$

Note that this value is slightly higher than $c_{h}$, in accord with the prediction from the sum rules and the analytic argument, and the difference between the two $\left(c_{d}-c_{h} \sim 0.002\right)$ is slightly smaller than the one that is obtained from the sum rules.

\section{Effect of the working temperature}

Up to now we have considered quenches to a zero working temperature. In this section we investigate the effect of having a finite temperature on the dynamics.

The arguments in Sec. IV rely on the $T=0$ Allen-Cahn equation (29). Temperature fluctuations have a twofold effect. On the one hand they generate equilibrium thermal domains that are not related to the coarsening process. On the other hand they roughen the domain walls thus opposing the curvature driven growth and slowing it down.

Renormalization group treatments of domain growth dynamics [25] have led to the idea that a $T=0$ fix point controls the domain growth for all $T<T_{c}$, i.e., that thermal fluctuations are irrelevant to the asymptotic dynamics of the ordering system, their contribution being limited primarily to the renormalization of temperature-dependent prefactors.

For the distribution of domain areas and hull enclosed areas, one may expect that once equilibrium thermal domains are subtracted-hulls and domains associated to the coarsening process are correctly identified - the full temperature dependence enters only through the values of $\lambda_{h}$ and $\lambda_{d}$, which set the time scale.

The first step then is to identify the temperature dependence of the parameter $\lambda_{d}$. The simplest and most direct way to do this is to use the scaling hypothesis and analyze the behavior of the spatial correlation

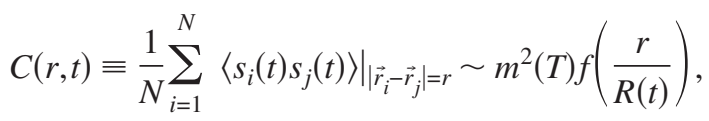

where $m(T)$ is the equilibrium magnetization density and $a$ $\ll r \ll L$ and $t_{0} \ll t$. Using $R(t) \sim\left[\lambda_{d}(T) t\right]^{1 / 2}$, the $T$ dependence of $\lambda_{d}$ can be estimated either by collapsing all curves or by studying the value of $r$ at which $C(r, t)=1 / 2$. The resulting
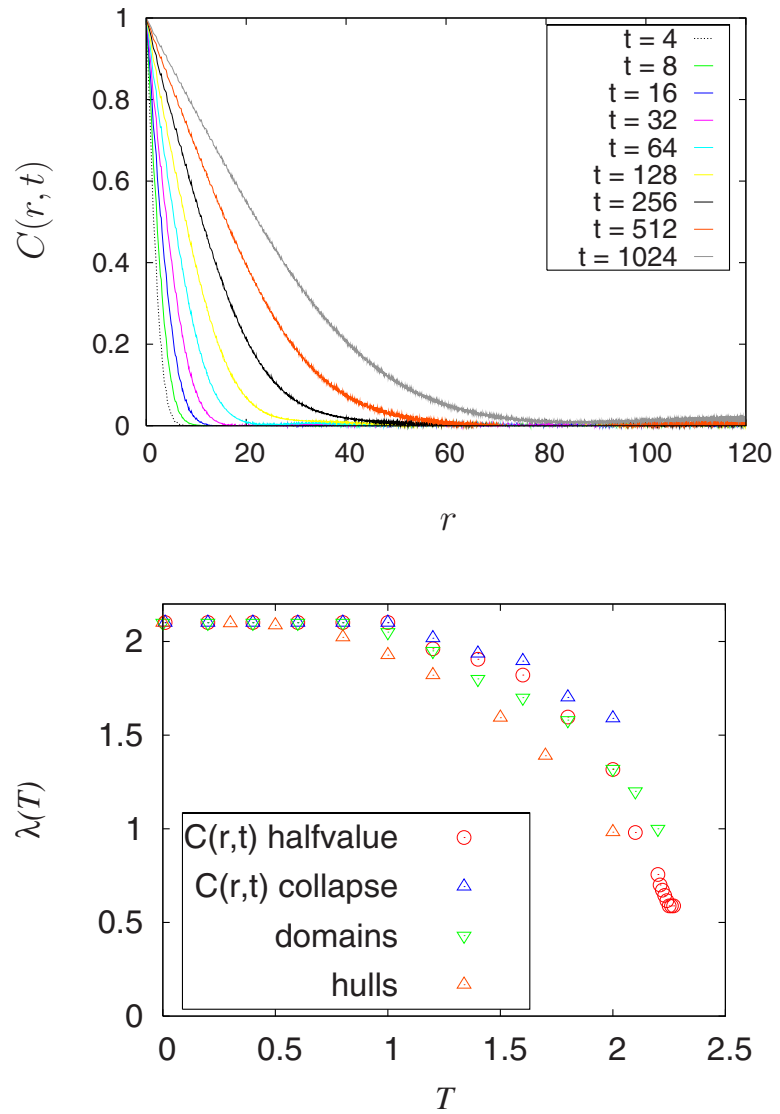

FIG. 17. (Color online) Top panel: spatial decay of the equaltime correlation, Eq. (59), at fixed $T=0$ and several different times $t$. Bottom panel: the $T$ dependence of the parameters $\lambda_{d, h}$. Two sets of data points are extracted from the analysis of the correlations shown in the top panel. The data named half value are obtained from $C(r, t)=1 / 2$ and the data named collapse from collapsing the curves on the range $r \gg a$. The two other sets are obtained from fitting $n_{d, h}(A, t)$ with $\lambda_{d, h}(T)$ as free parameters.

$\lambda_{d}(T)$ obtained using these two prescriptions is shown in Fig. 17. $\lambda_{d}(T)$ is a monotonically decreasing function of temperature, starting at $\lambda_{d}(T=0)=2.1$ and falling off to zero at $T_{c}$. These results are consistent with the evaluation of $\lambda_{d, h}(T)$ from the analysis of $n_{d, h}(A, t)$, see below, and it is at variance with what was previously presented in Ref. [26].

Assuming that $\lambda_{d}$ vanishes at $T_{c}$ one can derive the way in which it does with a simple argument [27]. We require that the coarsening law for coarsening below $T_{c}$, namely $R(t)$ $\sim[\lambda(T) t]^{1 / 2}$, match critical coarsening at $T_{c}$, viz. $R(t) \sim t^{1 / z}$ with $z$ the dynamic exponent, for $T \rightarrow T_{c}$. Near (but just below) $T_{c}$ the coarsening length grows as $\xi^{-a}(T) t^{1 / 2}$ as long as $R(t) \gg \xi(T)$ with $\xi(T)$ the equilibrium correlation length. For $R(t)$ comparable with $\xi(T)$, this has to be modified by a function of $R(t) / \xi$ and, since $R(t) \sim t^{1 / z}$ at $T_{c}$, we can write

$$
R(t) \sim \xi^{-a}(T) t^{1 / 2} f\left(\frac{t}{\xi^{2}(T)}\right) .
$$

In the limit $\xi(T) \rightarrow \infty$, the $\xi$ dependence must drop out. In order to cancel the time dependence at large times, one needs 


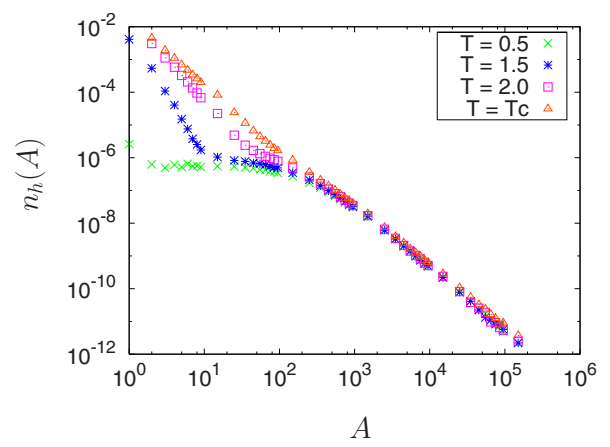

FIG. 18. (Color online) The number density of hull enclosed areas after $t=128 \mathrm{MCs}$ at the working temperatures $T=0.5,1.5,2$ and $T=T_{c}$.

$f(x) \sim x^{1 / 2}$ for $x \rightarrow \infty$. This yields $R(t) \sim t^{1 / z}$, which fixes the exponent " $a$ " as $a=(2-z) / 2$, giving $\lambda(T) \sim \xi^{-2 a}(T) \sim\left(T_{c}\right.$ $-T)^{\nu(z-2)}$. Inserting the exact value $\nu=1$ and the numerical value $z=2.15(2)[28]$ implies

$$
\lambda_{d}(T) \sim\left(T_{c}-T\right)^{0.15} .
$$

Note that we are matching two nonequilibrium growth laws - the one below $T_{c}$ and the one at $T_{c}$ - not an equilibrium and a nonequlibrium one. The data in Fig. 17 are still far from the critical region where this small power-law decay should show up.

Finite working temperatures also affect the distribution of domain areas. In Fig. 18 the raw data at $t=128 \mathrm{MCs}$ is shown for four working temperatures. Upward deviations with respect to the result of zero working temperature are prominent in the small-area regions of the figure, and increase with temperature.

In Fig. 19 (a) we display the raw data at the working temperature $T=1.5$, for several times. Notice that although the curves move downwards, the small areas region becomes time independent. This region also fails to collapse (b) with the proposed scaling using the temperature dependent values of $\lambda_{d}(T)$. The reason is that the distribution counts thermal equilibrium domains, that is to say, fluctuations that are present in an equilibrated sample at the working temperature, but are not due to the coarsening process. Thus, these fluctuations should be identified and eliminated from the statistics. We tried to apply the method introduced by Derrida [2], and extended by Hinrichsen and Antoni [29], to eliminate thermal domains, but the results were not satisfactory, as not all of them could be eliminated. Thus, instead of removing each thermal domain, we tried to directly remove their contribution to the distributions by simulating samples in equilibrium at the working temperature, starting with a fully magnetized state, and computing the number density of thermal domain areas. These data are shown with green data points in Figs. 19 and 20. Surprisingly enough, thermal fluctuations generate areas that are larger than one would have naively expected. Equilibrium arguments suggest that the averaged area of thermally generated domains scale as $A_{T}$ $\sim \xi^{2}(T)$ with $\xi(T) / p_{0} \sim f^{-}\left(1-T / T_{c}\right)^{-\nu}, \nu=1$, and $f^{-}=0.18$ [30]. This estimate yields, for example, $A_{T} \sim 4 A_{0}$ at $T=1.5$.

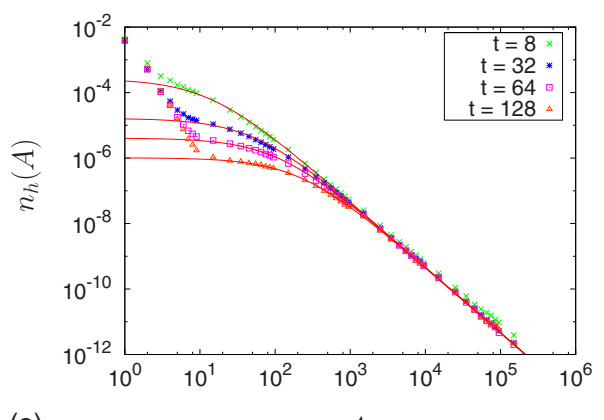

(a)

A

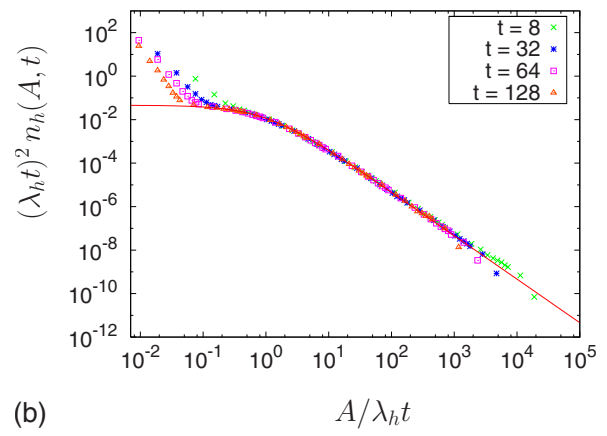

FIG. 19. (Color online) The number density of hulls for $T$ $=1.5$ after different times (a) and the scaling of these data points (b).

In equilibrium at this temperature the average size of the domains found numerically is $\left\langle A_{T}\right\rangle \sim 1.5 A_{0}$. However, the probability distribution of thermal areas has a non-negligible weight - as compared to the one of coarsening domainsthat goes well beyond this value. For example, in Figs. 19 and 20 we see that the crossover between the thermal area distribution and the coarsening area distribution occurs at $A \sim 10 A_{0}$.

In Fig. 20 we also present data for the dynamic distribution at three different times, and compared with the analytic prediction using $\lambda_{d}(T)$ estimated from the analysis of the global spatial correlation, see Fig. 17. We conclude that the agreement between analytic prediction and numerical results is very good in the region in which the thermal domains are

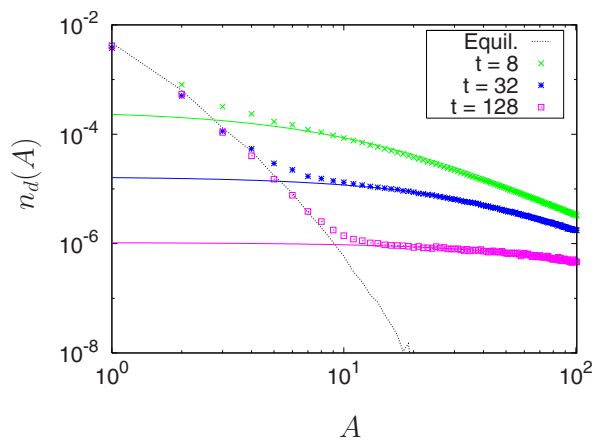

FIG. 20. (Color online) The contribution of thermal domains obtained by simulating an equilibrated sample at the working temperature $T=1.5$, along with the evolution of the distribution of domain sizes after a quench to the same temperature. 


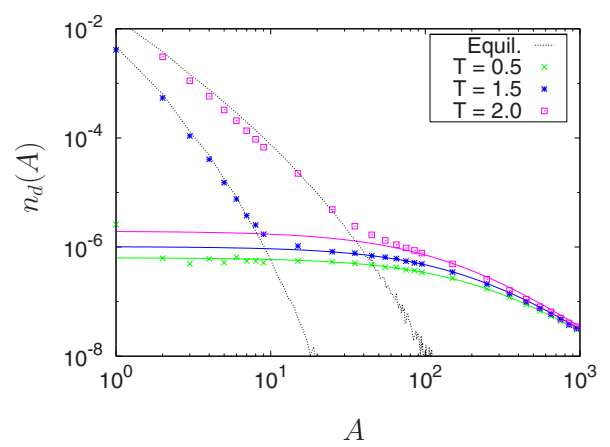

FIG. 21. (Color online) Zoom on the number density of domain areas at $t=128 \mathrm{MCs}$ at three working temperatures given in the key. The black lines are the equilibrium distributions at $T=1.5$ and $T$ $=2$ and the other lines (pink, blue, and green) represent our analytic prediction for the coarsening areas.

subdominant, i.e., when the blue dynamic curves deviate from the green equilibrium one, indeed the regime in which the analytic calculation is expected to apply.

One can also use the results in Fig. 21 to estimate the value of $\lambda_{d}(T)$. Indeed, a fit of the numerical data for areas larger than the value at which the equilibrium thermal contribution (green points) deviates from the dynamic one, yields the values of $\lambda_{d}(T)$ [and $\lambda_{h}(T)$ ] shown in Fig. 17. This analysis allows us to extract independent predictions for $\lambda_{d}(T)$ and $\lambda_{h}(T)$. We find that the qualitative $T$ dependence is the same. As regards the absolute values, the numerical data yield $\lambda_{h}(T) \leqslant \lambda_{d}(T)$ on the whole range. Note that the sum rules suggested $\lambda_{d}=\lambda_{h}$ and the analytic prediction $\lambda_{d}=\lambda_{h}$ $+\mathcal{O}\left(c_{h}\right)$.

\section{STATISTICS OF PERIMETERS AND FRACTAL PROPERTIES}

The analytic argument described in Sec. IV can be extended to study the distribution of domain wall lengths or perimeters. In this section we present the analytic prediction for this function together with numeric results that confirm it. We study two types of domain boundaries: those associated to the hulls and those associated to the domains that is to say that include external and internal perimeters. In the simulations we define the length of the boundary as the number of broken bonds.

\section{A. Initial conditions}

\section{Equilibrium at $T_{0}=T_{c}$}

In equilibrium we find numerically that the domain areas and their corresponding boundaries are related by (see Fig. 22 where the scatter plots have been averaged to make the trend clearer)

$$
A_{h} \sim c_{h}^{(i)} p^{\alpha_{h}^{(i)}}, \quad \text { with } \alpha_{h}^{(i)} \sim 1.47 \pm 0.1,
$$

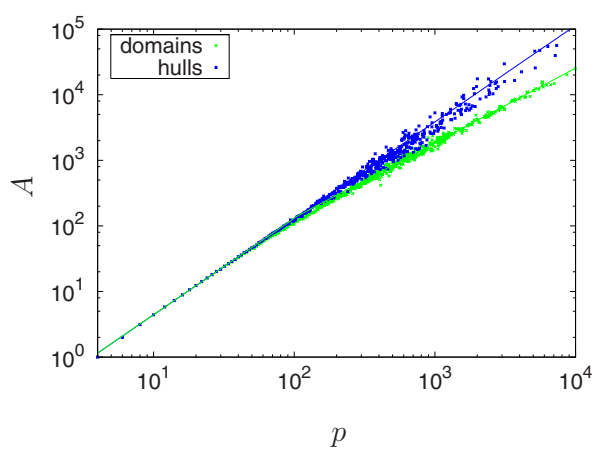

FIG. 22. (Color online) Relation between areas and perimeters in equilibrium at $T_{0}=T_{c}$.

$$
A_{d} \sim \begin{cases}c_{d}^{(i>)} p^{\alpha_{d}^{(i>)}}, & \alpha_{d}^{(i>)} \sim 1.14 \pm 0.1 \text { for } p \gtrsim 50, \\ c_{d}^{(i<)} p^{\alpha_{d}^{(i<)}}, & \alpha_{d}^{(i<)} \sim 1.47 \pm 0.1 \text { for } p \lesssim 50,\end{cases}
$$

in the whole range of variation. Note that the longest lengths, $p \approx 10^{3}-10^{4}$ may be affected by finite size effects given that the linear size of the simulating box is $L=10^{3}$. The spanning clusters are not counted (note that their perimeters would be severely under estimated due to the periodic boundary conditions). The exponent $\alpha_{d}^{(i<)} \sim 1.47 \pm 0.1$ is consistent with the result in [18] mentioned in Sec. II C. The constants take the values $c_{h}^{(i)}=0.15, c_{d}^{(i<)}=0.15$, and $c_{d}^{(i>)}=0.70$. The difference between the small and large $p$ regimes in the relation between areas and perimeters for the domains is due to the existance of holes in the large structures. The small domains and hulls are just the same objects because the former do not have holes within.

Numerically, we find that the number densities of hull and domain lengths at critical Ising conditions are (see Fig. 23)

$$
\begin{gathered}
n_{h}(p, 0) \sim p^{-\zeta_{h}} \quad \text { with } \zeta_{h} \sim 2.48 \pm 0.05, \\
n_{d}(p, 0) \sim \begin{cases}p^{-\zeta_{d}}, & \zeta_{d}^{>} \sim 2.17 \pm 0.05 \text { for } p \gtrsim 50, \\
p^{-\zeta_{d}^{<}}, & \zeta_{d}^{<} \sim 2.48 \pm 0.05 \text { for } p \lesssim 50 .\end{cases}
\end{gathered}
$$

The value of $\zeta_{h}$ is to be compared to the analytic result $\zeta_{h}$ $=27 / 11 \approx 2.454[10]$. It is interesting to notice that the dis-

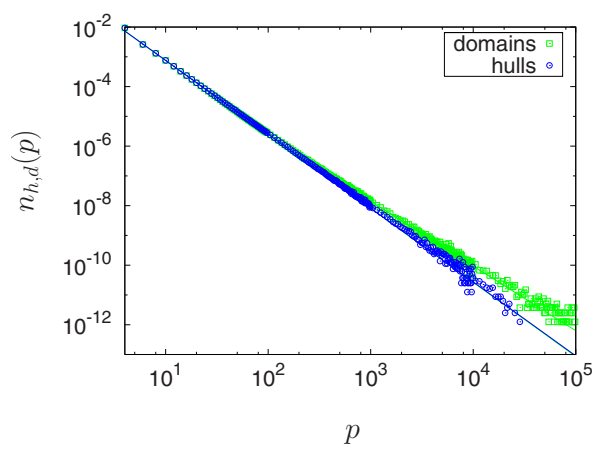

FIG. 23. (Color online) Distribution of domain and hull lengths in equilibrium at $T_{0}=T_{c}$. 


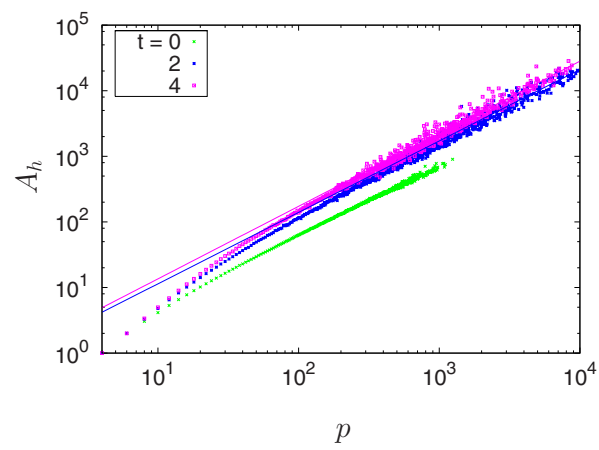

FIG. 24. (Color online) Relation between areas and perimeters in equilibrium at $T_{0} \rightarrow \infty$ and after a few time steps when critical percolation is approximatively reached.

tribution of domain lengths is not a single power law in constrast to the distribution of domain areas.

\section{Equilibrium at $T_{0}=\infty$}

After a few time steps evolving at $T=0$ from the infinite temperature initial condition, we reach critical percolation conditions.

In Fig. 24, we show the area perimeter relation for hulls, in equilibrium at $T_{0} \rightarrow \infty$ and after a few time steps. The analysis of this figure and the corresponding one for domains yields

$$
\begin{gathered}
A_{h} \sim c_{h}^{\left(i^{\prime}\right)} p^{\alpha_{h}^{\left(i^{\prime}\right)},}, \quad \text { with } \alpha_{h}^{\left(i^{\prime}\right)} \sim 1.12 \pm 0.1, \\
A_{d} \sim \begin{cases}c_{d}^{\left(i^{\prime}>\right)} p^{\alpha_{d}^{\left(i^{\prime}>\right)}}, & \alpha_{d}^{\left(i^{\prime}>\right)} \sim 1.01 \pm 0.1 \text { for } p \gtrsim 50, \\
c_{d}^{\left(i^{\prime}<\right)} p^{\alpha_{d}^{\left(i^{\prime}<\right)}}, & \alpha_{d}^{\left(i^{\prime}<\right)} \text { for } p \lesssim 50 .\end{cases}
\end{gathered}
$$

The constants take the values $c_{h}^{\left(i^{\prime}\right)}=0.96$ and $c_{d}^{\left(i^{\prime}>\right)}=1.50$. The exponent $\alpha_{d}^{\left(i^{\prime}<\right)}$ cannot be determined numerically since critical percolation is not accessible exactly.

As we can see in Fig. 25, the initial weight of the number density at large values of the perimeter is lower than expected at critical percolation. In a few time steps long perim-

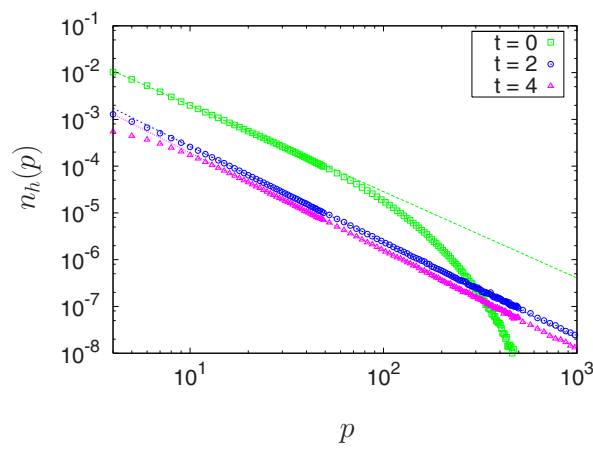

FIG. 25. (Color online) The number density of hull lengths in equilibrium at $T_{0} \rightarrow \infty$ labelled as $t=0$ and the same quantity evaluated at two later times after evolution at zero working temperature. The lines are $n_{h} \sim p^{-2.05}$; see the text for a discussion. eters develop and the weight reaches the asymptotic power law at large values of $p$ while it looses weight at small values of $p$. This effect is the same as the one observed in the study of the initial and early times number density of areas, see the discussion in Sec. V A and Fig. 5. For the hull and domain length distributions one finds

$$
\begin{gathered}
n_{h}(p, 0) \sim p^{-\zeta_{h}^{\prime}} \quad \text { with } \zeta_{h}^{\prime} \sim 2.12 \pm 0.05, \\
n_{d}(p, 0) \sim \begin{cases}p^{-\zeta_{d}^{\prime>}}, & \zeta_{d}^{\prime>} \sim 2.01 \pm 0.05 \text { for } p \gtrsim 50, \\
p^{-\zeta_{d}^{<}}, & \zeta_{d}^{<} \text {for } p \lesssim 50 .\end{cases}
\end{gathered}
$$

The analytical result for the hull exponent in critical percolation is $\zeta_{h}^{\prime}=15 / 7 \approx 2.14$ [11].

\section{General comments on both initial cases}

It is interesting to note that the exponents characterizing the number density of perimeter lengths at the two initial conditions are significantly different. They are approximately equal to 2.5 at $T_{c}$ and 2 at $T_{0} \rightarrow \infty$. This is to be contrasted with the behavior of the area number densities for which the exponents were identical for hull enclosed areas and very close indeed for domains.

The exponents $\alpha$ and $\zeta$ are linked by the fact that each hull enclosed area or domain area is in one-to-one relation to its own boundary. Thus, $n_{h}(A, 0) d A=n_{h}(p, 0) d p$ and one finds

$$
n_{h}(p, 0) \sim \frac{c_{h} \alpha_{h}^{(i)}}{c_{h}^{(i)}} p^{-1-\alpha_{h}^{(i)}},
$$

which implies

$$
\zeta_{h}=1+\alpha_{h}^{(i)} .
$$

These conditions are also satisfied for the primed $\left(T_{0} \rightarrow \infty\right)$ quantities. Within our numerical accuracy these relations are respected, for instance,

$$
\begin{array}{cl}
\zeta_{h}^{\prime} \sim 2.12, & \alpha_{h}^{\prime(i)} \sim 1.12, \quad T_{0} \rightarrow \infty, \\
\zeta_{h} \sim 2.48, & \alpha_{h}^{(i)} \sim 1.47, \quad T_{0}=T_{c} .
\end{array}
$$

Similarly, for domain areas and domain boundaries one obtains

$$
n_{d}(p, 0) \sim \frac{c_{d} \alpha_{d}^{(i)}}{c_{d}^{(i)}} p^{-1-(\tau-1) \alpha_{d}^{(i)}},
$$

therefore,

$$
\zeta_{d}=1+(\tau-1) \alpha_{d}^{(i)} .
$$

These relations are satisfied for both $\alpha_{d}^{(i>)}$ and $\alpha_{d}^{(i<)}$ as well as for primed $\left(T_{0} \rightarrow \infty\right)$ quantities. They are respected by our measures.

The main sources of error in the determination of the exponents and the constants in the study of the initial conditions are the following: (i) statistical errors, although we have a rather good sampling; (ii) the choice of the large area-perimeter limit that is not perturbed by finite size ef- 


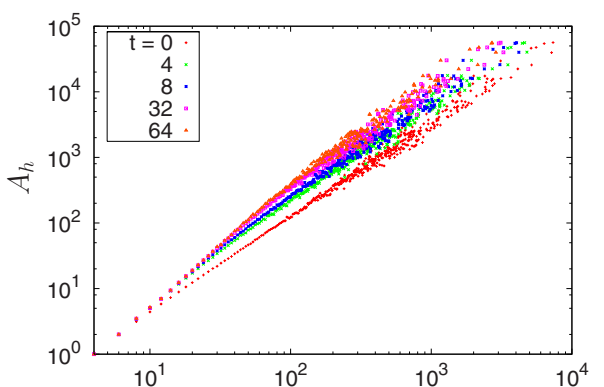

(a)

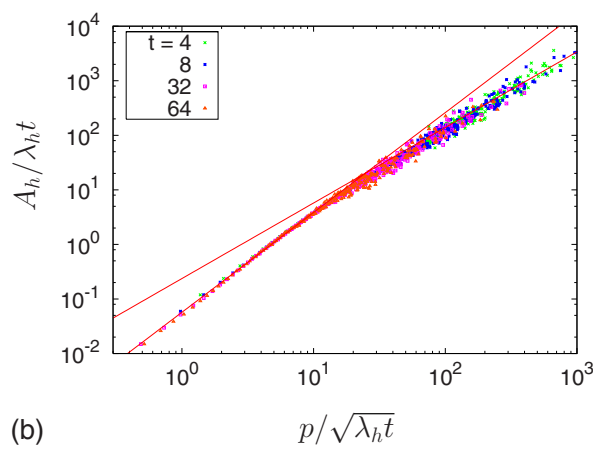

FIG. 26. (Color online) Time evolution of the hull enclosed area vs perimeter relation for $T_{0}=T_{c}$ and different times indicated in the legend.

fects, and (iii) the fact that the $T_{0} \rightarrow \infty$ initial condition is not exactly at critical percolation. We estimated the magnitude of the error to be \pm 0.1 in the $\alpha$ exponents, and \pm 0.05 in the $\zeta$ exponents, which correspond to, roughly, less than $10 \%$ in both cases. Within this level of accuracy, the relations between exponents (65) and (63) are satisfied.

\section{B. Time evolution at zero temperature}

\section{Hulls}

After a quench from $T_{0}=T_{c}$, the hull enclosed areas and their corresponding perimeters, during coarsening at zero temperature, obey the scaling relations (see Fig. 26)

$$
\frac{A}{\lambda_{h} t} \sim \eta_{h}\left(\frac{p}{\sqrt{\lambda_{h} t}}\right)^{\alpha_{h}},
$$

with

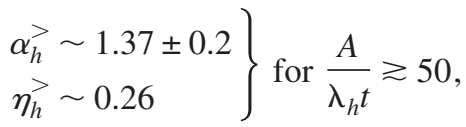

and

$$
\left.\begin{array}{l}
\alpha_{h}^{<} \sim 1.83 \pm 0.2 \\
\eta_{h}^{<} \sim 0.06
\end{array}\right\} \text { for } \frac{A}{\lambda_{h} t} \lesssim 10,
$$

and, after a quench from $T_{0} \rightarrow \infty$,

$$
\frac{A}{\lambda_{h} t} \sim \eta_{h}^{\prime}\left(\frac{p}{\sqrt{\lambda_{h} t}}\right)^{\alpha_{h}^{\prime}}
$$

with

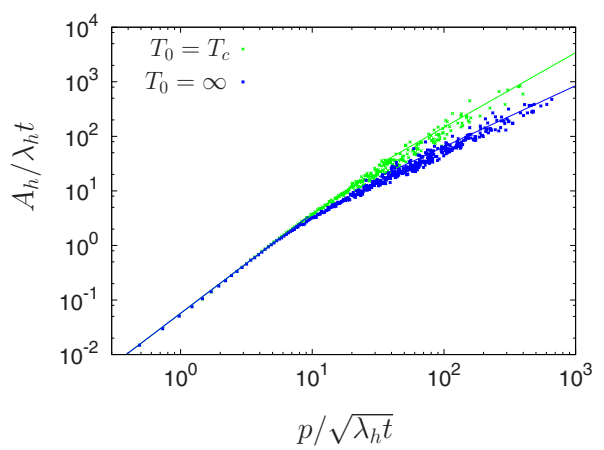

FIG. 27. (Color online) Hull enclosed area vs perimeter relation at time $t=32 \mathrm{MCs}$ from two different initial conditions.

$$
\left.\begin{array}{l}
\alpha_{h}^{\prime>} \sim 1.12 \pm 0.2 \\
\eta_{h}^{\prime>} \sim 0.38
\end{array}\right\} \text { for } \frac{A}{\lambda_{h} t} \geq 50
$$

and

$$
\left.\begin{array}{l}
\alpha_{h}^{\prime<} \sim 1.83 \pm 0.2 \\
\eta_{h}^{\prime<} \sim 0.057
\end{array}\right\} \text { for } \frac{A}{\lambda_{h} t} \lesssim 10 .
$$

We note that the relation between area and perimeter exhibits two distinct regimes. During the coarsening process a characteristic scale $A^{*}(t) \sim \lambda_{h} t$ develops such that domains with area $A>A^{*}$ have the same exponent as in the initial condition (structures that are highly ramified with $\alpha$ smaller than two) and domains with $A<A^{*}$ are regular $(\alpha \sim 2)$ (as shown in Fig. 27 the structure of these small domains does not depend on the initial condition). This phenomenon is reminiscent of an unroughening transition occurring at a velocity $\lambda_{h}$. The same features were observed by Grest and Srolovitz [31] and Fialkowski and Holyst [32] in the study of the domain fractal dimension during coarsening.

The hull structures of any size do not have holes, therefore the crossover we see is of pure dynamical origin, contrasting the idea presented in [33], where the crossover in the domains where explained by only geometrical reasons.

Note that we estimated the error in the exponents $\alpha$ to be \pm 0.2 and thus more important than in the analysis of the initial conditions. The reason is that the crossover from the small area to the large area regime is not sufficiently sharp and the choice of the fitting interval introduces an additional source of error. Indeed, note that in Eqs. (66) and (69) we did not use the intermediate regime $10 \leqslant \frac{A}{\lambda_{h} t} \leqslant 50$ to fit the power laws.

In analogy with the derivation in Sec. IV for the timedependent number density of domain areas, the timedependent number densities of hull and domain-wall lengths are given by

$$
n_{h, d}(p, t)=\int d p_{i} \delta\left[p-p\left(t, p_{i}\right)\right] n_{h, d}\left(p_{i}, t_{i}\right)
$$

with $n_{h, d}\left(p_{i}, t_{i}\right)$ the initial condition and $p\left(t, p_{i}\right)$ the perimeter length of a boundary at time $t$ that had initial length $p_{i}$ at time $t_{i}$. 
Let us here discuss the hull lengths. In this case one can simply use the exact number density of hull enclosed areas, $n_{h}(A, t) \sim c_{h} /\left(A+\lambda_{h} t\right)^{2}$ for, say, $T_{0}=T_{c}$ and Eq. (66) to relate time-dependent areas to their perimeters on the two regimes of large and small areas. After a little algebra one derives

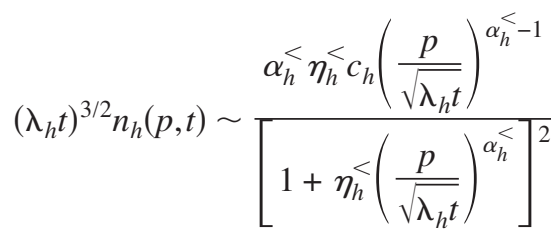

for small areas, $A / \lambda_{h} t<10$, and

$$
\begin{gathered}
\left(\lambda_{h} t\right)^{3 / 2} n_{h}(p, t) \sim \\
{\left[1+\eta_{h}^{>}\left(\frac{p}{\sqrt{\lambda_{h} t}}\right)^{\alpha_{h}} c_{h}\left(\frac{p}{\sqrt{\lambda_{h} t}}\right)^{\alpha_{h}^{>}-1}\right.}
\end{gathered}
$$

for large areas $A / \lambda_{h} t>50$. Note that these expressions satisfy scaling-see Eq. (11). Interestingly, the scaling function, $f_{<}(x)=x^{\alpha_{h}^{<}-1} /\left(1+\eta_{h}^{<} x^{\alpha_{h}^{<}}\right)^{2}$ with $x=p / \sqrt{\lambda_{h} t}$ reaches a maximum at

$$
x_{\max }=\left(\frac{\alpha_{h}^{<}-1}{\eta_{h}^{<}\left(\alpha_{h}^{<}+1\right)}\right)^{1 / \alpha_{h}^{<}}
$$

and then falls off to zero as another power law. There is then a maximum at a finite and positive value of $p$ as long as $\alpha_{h}^{<}>1$, that is to say, in the regime of not too large areas. The numeric evaluation of the right-hand side yields $x_{\max }$ $=p_{\max } /\left(\sqrt{\lambda_{h} t}\right) \sim 3$ which is in the range of validity of the scaling function $f_{<}$. The behavior of the time-dependent perimeter number density for long perimeters is controlled by Eq. (74) that falls off as a power law $f_{>}(x) \sim x^{-\left(1+\alpha_{h}\right)}$. Although the function $f_{>}$also has a maximum, this one falls out of its range of validity.

Above we used the critical Ising parameters. The results after a quench from $T_{0} \rightarrow \infty$ follow the same functional form with the corresponding primed values of $\alpha$ and $\eta$ and $c_{h}$ $\rightarrow 2 c_{h}$.

The power law describing the tail of the number density of long perimeters is the same as the one characterizing the initial distribution, since $\alpha_{h}^{>}=\alpha_{h}^{(i)}$ and then $1+\alpha_{h}^{>}=\zeta_{h}$. Therefore, the decay of the time-dependent number density at long perimeters after a quench from $T_{0}=T_{c}$ and $T_{0} \rightarrow \infty$ are distinguishably different with $\zeta_{h} \approx 2.5$ and $\zeta_{h}^{\prime} \approx 2$. This is to be contrasted with the small difference in the area number densities that fall with two power laws that are so close (powers of 2 and 2.05) that are impossible to distinguish numerically.

In Fig. 28, panels (a) and (b), we display the timedependent perimeter number densities for a system evolving at zero temperature after a quench from $T_{0}=T_{c}$ and $T_{0} \rightarrow \infty$, respectively. Notice that the perimeter length definition we are using on the lattice can only take even values and thus when constructing the histogram we have to take into account the extra factor of 2 in the binning.

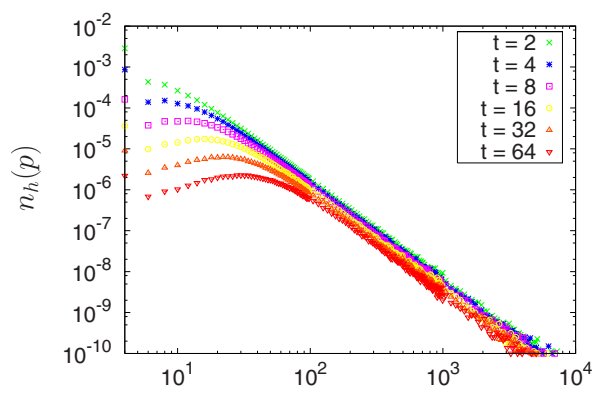

(a)

$p$

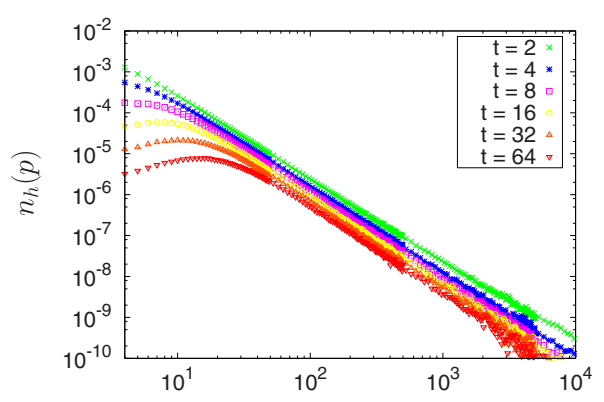

(b)

$p$

FIG. 28. (Color online) The time-dependent number density of perimeters evolving at $T=0$ from an initial condition at $T_{0}=T_{c}$ (a) and $T_{0} \rightarrow \infty$ (b). Note that the time-dependence is visible in the whole range of values of $p$ (while in the area number densities the large area tails were very weakly dependent on time, see Fig. 7).

In Fig. 29 we display the scaling plot of the number density of hull lengths and we compare it to the analytic prediction (73) and (74). The data are in remarkably good agreement with the analytic prediction; the lines represent the theoretical functional forms for long and short lengths, and describe very well the two limiting wings of the number density. The maximum is located at a value that is in agreement with the prediction, Eq. (75).

\section{Domains}

We studied the relation between domain areas and their corresponding perimeters during coarsening at zerotemperature finding that the scaling forms

$$
\frac{A}{\lambda_{d} t} \sim \eta_{d}\left(\frac{p}{\sqrt{\lambda_{d} t}}\right)^{\alpha_{d}}
$$

with

$$
\left.\begin{array}{l}
\alpha_{d}^{>} \sim 1.16 \\
\eta_{d}^{>} \sim 0.63
\end{array}\right\} \text { for } \frac{A}{\lambda_{d} t} \geq 50
$$

and

$$
\left.\begin{array}{l}
\alpha_{d}^{<} \sim 1.83 \\
\eta_{d}^{<} \sim 0.057
\end{array}\right\} \text { for } \frac{A}{\lambda_{d} t} \leqslant 10,
$$

after a quench from $T_{0}=T_{c}$ and 

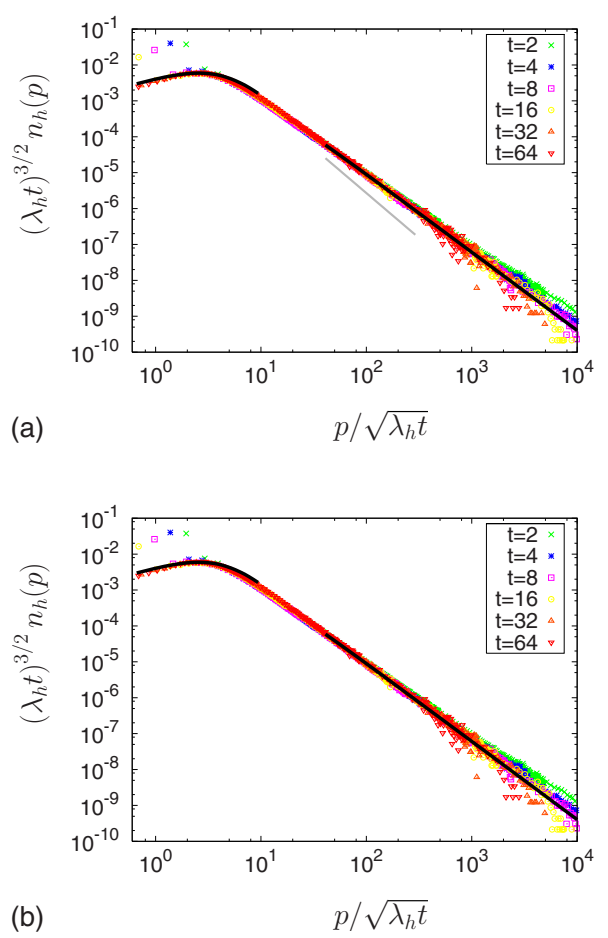

FIG. 29. (Color online) Scaling of the time-dependent number density of hull lengths evolving at $T=0$ from an initial condition at $T_{0}=T_{c}$ (a) and $T_{0} \rightarrow \infty$ (b). The solid black lines represent the theoretical prediction valid for $A / \lambda_{h} t<10$ and for $A / \lambda_{h} t>50$. The agreement between theory and numerical data is again very impressive. The small grey line in panel (a) represents the slope in panel (b), showing that in contrast with domain size distribution, perimeter distribution is very sensitive to initial conditions. The isolated data points that lie above the scaling function correspond to reversed, isolated spins within a bulk of the opposite sign that give rise to a perimeter length $p=4$ (four broken bonds). The area number densities also showed this anomalous behavior for $A=1$.

$$
\frac{A}{\lambda_{d} t} \sim \eta_{d}^{\prime}\left(\frac{p}{\sqrt{\lambda_{d} t}}\right)^{\alpha_{d}^{\prime}}
$$

with

$$
\left.\begin{array}{l}
\alpha_{d}^{\prime>} \sim 1.01 \\
\eta_{d}^{\prime>} \sim 0.52
\end{array}\right\} \text { for } \frac{A}{\lambda_{d} t} \geq 50
$$

and

$$
\left.\begin{array}{l}
\alpha_{d}^{\prime<} \sim 1.83 \\
\eta_{d}^{\prime<} \sim 0.06
\end{array}\right\} \text { for } \frac{A}{\lambda_{d} t} \leqslant 10
$$

after a quench from $T_{0} \rightarrow \infty$.

These results are shown in Fig. 30. Note that the large area results match the behavior of the initial conditions in both cases, and small domains are much more compact that the initial ones.

With the same line of argument exposed above we can analyze the statistics of the domain walls, that is to say, including external and internal perimeters. One finds basically
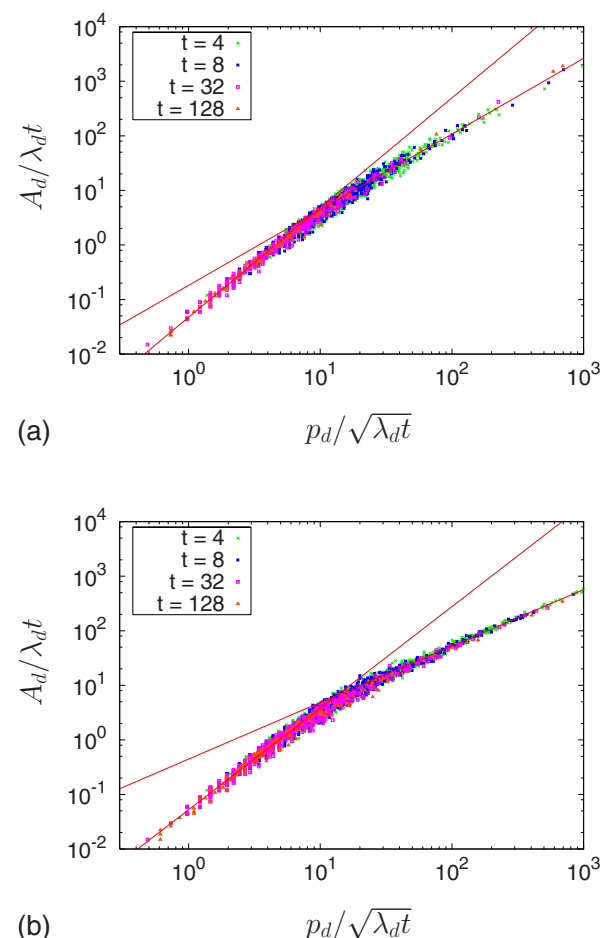

FIG. 30. (Color online) The time-dependent relation between the area and the perimeter of a cluster of aligned spins (domain) evolving at $T=0$ after a quench from $T_{0}=T_{c}$ (a) and $T_{0} \rightarrow \infty$ (b). In both cases $\lambda_{d}=2.1$.

the same results as for the hulls; for critical Ising initial conditions

$$
\left(\lambda_{d} t\right)^{3 / 2} n_{d}(p, t) \sim \frac{\alpha_{d}^{<} \eta_{d}^{<} c_{d}\left(\frac{p}{\sqrt{\lambda_{d} t}}\right)^{\alpha_{d}^{<}-1}}{\left[1+\eta_{d}^{<}\left(\frac{p}{\sqrt{\lambda_{d} t}}\right)^{\alpha_{d}^{<}}\right]^{\tau}}
$$

for small areas and its obvious modification for large areas. For $T_{0} \rightarrow \infty$ one replaces $\eta_{d}$ and $\alpha_{d}$ by the primed quantities and $c_{d} \rightarrow 2 c_{d}$. The scaling analysis of the number density of domain-wall lengths is displayed in Fig. 31 for both initial conditions. Once again we find a very good agreement between the analytic predictions and the numerical data.

\section{Finite temperature evolution}

Once we analyzed the statistics of perimeters in the zerotemperature dynamics we focus on the effects of a finite working temperature. We briefly list the results below without presenting the data.

\section{The area-perimeter relations}

For large areas we find the same exponent as for zerotemperature coarsening that is also the initial condition exponent $\left(T_{0} \rightarrow \infty\right.$ or $\left.T_{0}=T_{c}\right)$. This is reasonable since the large structures are still "unaware" of the coarsening process and thus retain the form they had in the initial configuration. For 

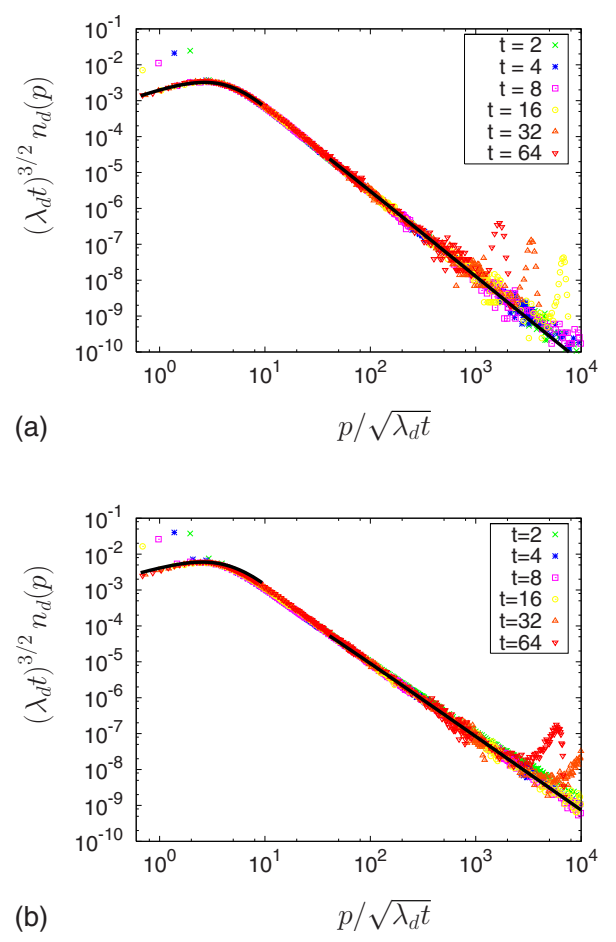

FIG. 31. (Color online) Scaling of the time-dependent number density of domain-wall lengths evolving at $T=0$ from an initial condition at $T_{0}=T_{c}$ (a) and $T_{0} \rightarrow \infty$ (b). The solid black lines represent the theoretical prediction valid for $A / \lambda_{h} t<10$ and for $A / \lambda_{h} t$ $>50$. The origin of the isolated data points is the same as in Fig. 29.

small areas, instead, we see domain walls roughening due to thermal agitation but it is hard to extract the value of the exponent $\alpha^{<}$with sufficient accuracy.

\section{Perimeter number densities}

The scaling of the perimeter number densities and the functional form for the scaling function predicted analytically describe the numerical data with high precision once the values of the exponents $\alpha$, the prefactors $\eta$, and the parameter $\lambda$ are modified to take into account thermal agitation (for absolute area values larger than $A \sim 10 A_{0}$, this value limits the range of areas where the effect of thermal fluctuations is larger than the ones of coarsening). The analytic prediction is very accurate in the region of small coarsening domains, $A / \lambda_{d, h} t<10$ and $A>10 A_{0}$ where the maximum is located, and in the region of large coarsening domains, $A / \lambda_{d, h} t>10$ and $A>10 A_{0}$, for both domains and hulls and the two initial conditions.

\section{CONCLUSIONS}

In this paper we studied the statistics and geometry of hull enclosed and domain areas and interfaces during the nonequilibrium dynamics of curvature driven pure coarsening in two dimensions. The analytical part of our work relies on the Allen-Cahn equation derived from the continuous GinzburgLandau field theory in two dimensions while the numerical part of it dealts with Monte Carlo simulations of the 2dIM. Our main results are the following: (i) We proved scaling of the various number densities studied.

(ii) We derived the exact number density of hull enclosed areas and hull lengths; we obtained approximate expressions for the number density of domain areas and domain-wall lengths.

(iii) The geometrical properties and distribution of the time-dependent large structures (by large we mean much larger than the average ones) are the ones of critical continuous percolation (for all initial conditions equilibrated at $T_{0}$ $>T_{c}$ ) and critical Ising (for $T_{0}=T_{c}$ ). The long interfaces retain the fractal geometry imposed by the equilibrium initial condition and the scaling function of all number densities decay as power laws.

(iv) Instead, small structures progressively become regular and the area perimeter relation is $A \sim p^{2}$.

(v) We took into account the effects of a finite working temperature by correctly eliminating purely thermal fluctuations and thus correctly identifying the coarsening structures. The temperature effect thus amounts to introducing the temperature dependence in the prefactor in the growth law, $R(t) \sim[\lambda(T) t]^{1 / 2} . \lambda(T)$ is a monotonically decreasing function of $T$ that vanishes at $T_{c}$.

It is important to stress that our analytic results rely on the use of the Allen-Cahn result for the velocity of an almost flat interface. Thus, they would be expected to hold only in a statistical sense and for large structures in the lattice model. Surprisingly, we found with numerical simulations that the number density area distributions in the 2dIM match the analytic predictions for very small structures, and even after a few MC steps evolution of a critical Ising initial condition for which rather rough interfaces exist.

Using the Allen-Cahn result and a variety of numerical measurements we verified the well-known result for pure coarsening with nonconserved order parameter: there is a characteristic growing length that increases in time as $t^{1 / 2}$. The mesoscopic analysis presented here allows us to demonstrate that the reason for the growth of the characteristic length is the disappearance of small structures.

Our analytic results hold only in two dimensions. In Appendix $\mathrm{B}$ we summarized the behavior of the distribution of domain lengths in the Ising chain. As expected from scaling arguments, this quantity scales with the typical domain length, $R(t) \sim t^{1 / 2}$ but the form of the scaling function is very different from the one in two dimensions: the pdf vanishes at zero scaling argument $(x=0)$, it then increases linearly to reach a maximum and then falls off to zero exponentially. On the other hand, we cannot extend the analytic argument to dimensions higher than two since the hull enclosed volumes no longer decrease with time in a manner that does not depend on their own volume [20].

This paper and [5] open the way to a number of related studies. For instance, it would be interesting to extend the analysis presented here to dynamic clusters of correlated spins (droplets), that are known to describe thermal fluctuations close to the transition (see $[34,35]$ for a review). These droplets are smaller than the geometric clusters in which they are embedded because some of the neighboring parallel spins are discarded (by a temperature-dependent criteria) for not being correlated (remember that even at infinite temperature, 
where all spins are uncorrelated, there are domains of parallel spins).

Two-dimensional coarsening with conserved order parameter is another problem that deserves a careful study along these lines.

In [5] we include a preliminary analysis of the hull and domain structure in the finite temperature dynamics of the bidimensional random bond ferromagnetic Ising model after a quench from infinite temperature. In such a disordered case a finite working temperature is necessary to help the interfaces depin from pinning centers in the quenched disordered potential through thermal activation. We first computed the typical domain radius that scales the time-dependent spatial correlation, $C(r, t) \sim f[r / R(t)]$, in the scaling regime. Due to the presence of quenched disorder $R(t)$ strongly depends on temperature and the strength of randomness and it is slower than the simple square root behavior of the pure Ising case. We then showed numerically that the number density of hull enclosed and domain areas scale as $R^{4}(t) n_{h, d}(A, t)$ $\sim g\left[A / R^{2}(t)\right]$ for areas satisfying $10^{-1} \lesssim A / R^{2}(t)$ and that are smaller than the cutoff set by finite size effects. The effect of a nontrivial typical radius $R(t)$ determined by the quenched disorder can be tested in the intermediate regime, say $10^{-1}$ $\lesssim A / R^{2}(t) \lesssim 10^{1}$, where the quality of the scaling plot is excellent. The scaling function $g(x)$ does not depend on the disorder strength satisfying the hyperscaling hypothesis [1]. For smaller areas, say $A / R^{2}(t) \lessgtr 10^{-1}$, the contribution of thermal domains with domain walls roughened by disorder is important. We shall give more details on the domain morphology of the quenched disordered coarsening problem in a separate publication [36].

These results give an idea of the richness and complexity of coarsening phenomena even in the absence of quenched randomness. We expect them to be of help in understanding the fluctuating dynamics of even more complex situations, such as spin-glasses and glassy problems [37], in which the mere existence of a domain growth of two competing equilibrium phases is not even established.

\section{ACKNOWLEDGMENTS}

A.J.B. and L.F.C. thank the Newton Institute at the University of Cambridge, UK, J.J.A. the LPTHE Jussieu, and A.S. and L.F.C. the Universidad Nacional de Mar del Plata, Argentina, for hospitality during the preparation of this work. A.S. and L.F.C. acknowledge financial support from SecytECOS Grants No. P. A01E01 and No. PICS 3172, A.S., J.J.A. and L.F.C. acknowledge financial support from CAPES-Cofecub research Grant No. 448/04. J.J.A. is also partially supported by the Brazilian agencies $\mathrm{CNPq}$ and FAPERGS. We thank S. Bustingorry and A. B. Kolton for useful discussions.

\section{APPENDIX A: ALGORITHM USED TO IDENTIFY HULL ENCLOSED AREAS}

In order to obtain the size of each hull in an $L \times L$ system, a biased walk along the interior border of each domain is performed, with the hull enclosed area being updated at each step. This algorithm is related to, but different from the Grossman-Aharony proposal.

Labelling. The $N$ sites are initially indexed from 0 to $N$ -1 (top-left=0) while the domains (geometric, ConiglioKlein, etc.) are identified and labelled by the HoshenKopelman algorithm [21]. By construction, all sites in each domain receive the (unique) label corresponding to the smaller index among its spins.

Starting point. The putative starting site for the walk is the spin whose index identifies the cluster. In some cases (for example, when the cluster crosses a border), it may not be the leftmost and/or topmost site, as it should in order to be counted correctly by the implementation of the algorithm below. Although such domains may be excluded from the statistics, for finite sizes the introduced bias is unacceptable. Thus, before starting, we try to find another site in the same cluster above or to the left of the original spin, where a new starting site may be found. Once this first site is correctly identified, we assign a height $y_{0}$.

The walk. From the starting point, we try to turn clockwise around the domain border. Viewed from the incoming direction, the attempted move is performed in the sequence: left, front, right, and backwards. For the sake of notation, we label the four directions with the indices shown in the Table I. Thus, for example, if the previous step was 0 , the attempted order will be $1,0,3$, and 2 .

The area. The first step sets the area to the value $A_{1}=y_{0}$ +1 . As the walk proceeds, both and area and the height are updated, $A_{i+1}=A_{i}+\Delta A$ and $y_{i+1}=y_{i}+\Delta y$ (in this order), where $\Delta A$ and $\Delta y$ depend also on the former direction (see Table I). At the end of the walk, when the departure site in reached, if the last direction is 1 , we increase $A$ by $-y$. Care should be taken when the starting point has right and bottom neighbors belonging to the same cluster (but not the bottom right diagonal), because in this case the walk should only be finished after the second time the starting point is visited.

\section{APPENDIX B: THE ONE-DIMENSIONAL CASE}

For the sake of comparison we present in this appendix results for the domain size distribution in the onedimensional Ising chain. The 1D ferromagnet with nearestneighbors interactions is given by the Hamiltonian

$$
\mathcal{H}=-J \sum_{i} s_{i} s_{i+1}
$$

where the spins are $s_{i}= \pm 1$ and $J>0$. The system evolves through heat bath dynamics, where each spin tries to align with its local field (with a temperature-dependent probability).

We measure the domain length distribution at time $t$, $n(\ell, t)(1 \leqslant \ell \leqslant L)$ after quenching the system from $T_{0} \rightarrow \infty$ to a final, working temperature, $T$. There is no finite static critical temperature and the system orders ferromagnetically only at $T=0$. At all finite temperatures a completely disordered initial condition starts evolving in a coarsening regime in which regions of finite length order and subsequently crosses over to equilibrium in the paramagnetic phase. Differently 
TABLE I. Incremental area contribution for each step during the oriented walk along the domain border. The values depend both on the present $(t)$ and former steps $(t-1)$. On the right we show the labelling of the four possible directions.

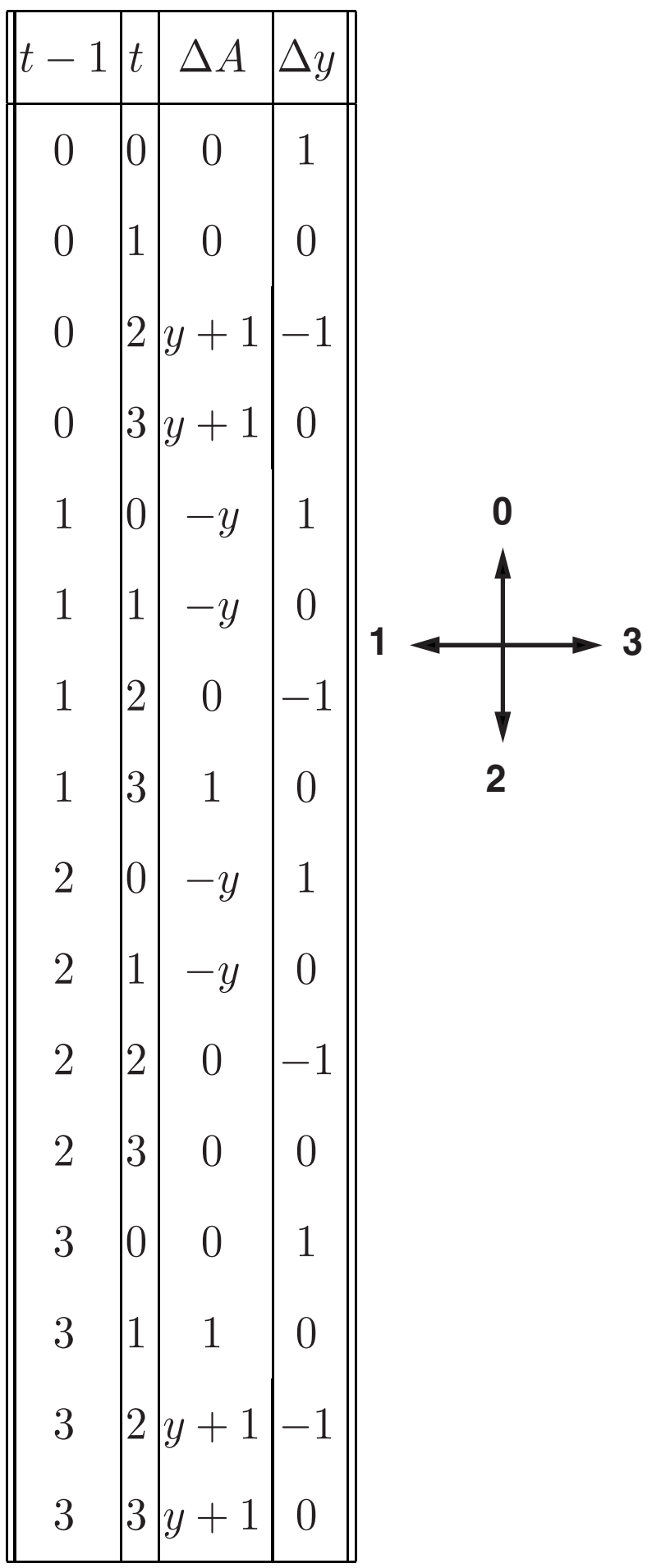

from the higher-dimensional case where domain growth is driven by interfacial tension, in $d=1$ coarsening is driven by the diffusion of domain walls and annihilation when they meet.

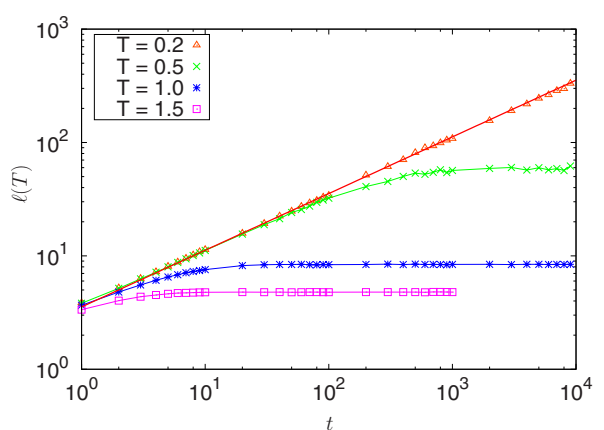

FIG. 32. (Color online) The average domain length at finite temperature, $\ell(t)=2 \sqrt{\pi t}$, independently of temperature in the domain-growth regime before saturation.

In the infinite temperature initial condition the spins are uncorrelated and $n(\ell, 0)=2^{-\ell}$, while in equilibrium, the normalized distribution is also exponential as it corresponds to the distribution of domains of a paramagnet in a field: $n(\ell, \infty)=r(1-r)^{\ell-1}$, with $r=[1+\exp (2 \beta)]^{-1}[38]$.

During the coarsening regime, the distribution of domain sizes obeys the scaling behavior

$$
n(\ell, t)=\langle\ell(t)\rangle^{-1} f\left(\frac{\ell}{\langle\ell(t)\rangle}\right)
$$

where the time-dependent average length is $\langle\ell(t)\rangle=2 \sqrt{\pi t}$ $[39,40]$, independently of temperature and saturates at $\langle\ell(\infty)\rangle=1 / r$ after a time that roughly grows as $\exp (4 / T)$, see Fig. 32. At zero working temperature the universal function $f(x)$ is given by [39-42]

$$
f(x)= \begin{cases}\pi x, & x \ll 1, \\ \exp (-A x+B), & x \gtrsim 1,\end{cases}
$$

where the constants are known exactly: $A=1.3062$ and $B$ $=0.597$ [40], see Fig. 33. Differently from higher dimensions, here as large clusters coalesce, there is no formation of small clusters (compared with the typical cluster). This fact

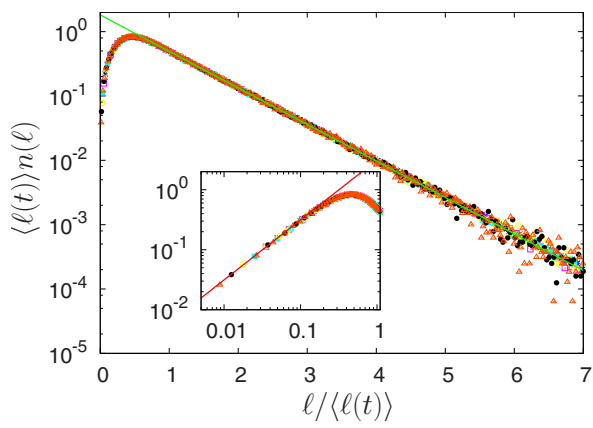

FIG. 33. (Color online) Collapsed distributions of the (rescaled) probability distribution of domain lengths after a quench to $T=0$ for $L=10^{5}$ and times $t=2^{3}, \ldots, 2^{9}$. All data collapse onto an universal curve whose tail is exponential [40]. Inset: the distribution for short length scales where the universal function is $f(x) \simeq \pi x$. Notice that the average domain length is different from the typical one. 


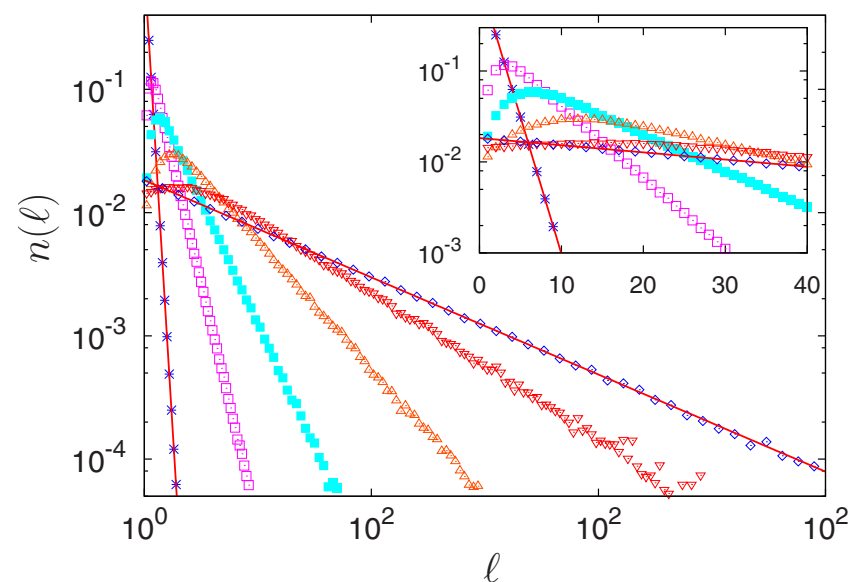

FIG. 34. (Color online) Domain length distribution at $T=0.5$ for increasing values of time (from left to right: $0,4,16,64,256$, and $\infty$. The initial, $n(\ell, 0)$, and final distributions, $n(\ell, \infty)$, are also indicated with solid lines (see text). The small $\ell$ region is shown in the inset.

is at the origin of the dip in the pdf close to $\ell /\langle\ell\rangle=0$ (see Fig. 34).

At finite working temperatures, because of the thermal fluctuations, small clusters are created at a constant

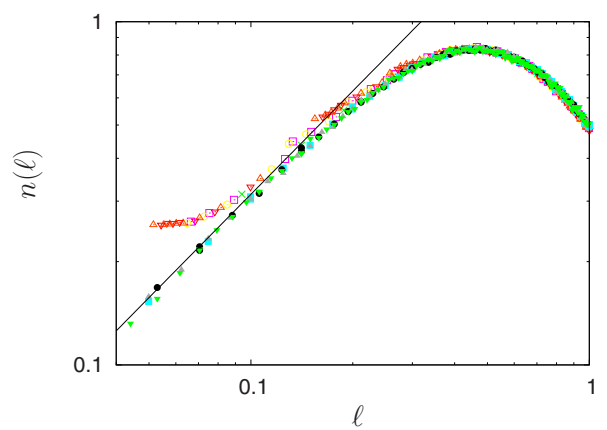

FIG. 35. (Color online) Temperature dependence of the domain length distribution at small lengths. Filled symbols are for $T=0$ while hollow ones are for $T=0.5$. The line is Eq. (B3) for small $x$.

temperature-dependent rate. As, say, one spin flips within a domain not only there is a new domain with length $\ell=1$, but the host domain has been cut in two pieces of relative much shorter length. Thermal agitation thus decreases the depth of the dip. When $x \gtrsim 1, f(x)$ is still well fitted by Eq. (B3). In the opposite regime, $x \ll 1$, instead thermal fluctuations diminish the dip and the behavior deviates from the linear scaling function, as can be seen in Fig. 35.
[1] See A. J. Bray, Adv. Phys. 43, 357 (1994), and references therein, for a review of coarsening phenomena.

[2] B. Derrida, Phys. Rev. E 55, 3705 (1997).

[3] J. A. Glazier, Phys. Rev. Lett. 70, 2170 (1993).

[4] R. D. MacPherson and D. J. Srolovitz, Nature (London) 446, 1053 (2007).

[5] J. J. Arenzon, A. J. Bray, L. F. Cugliandolo, and A. Sicilia, Phys. Rev. Lett. 98, 145701 (2007).

[6] J. Cardy and R. M. Ziff, J. Stat. Phys. 110, 1 (2003).

[7] D. Stauffer and A. Aharony, Introduction to Percolation Theory, 2nd ed. (Taylor \& Francis, London, 1994).

[8] A. L. Stella and C. Vanderzande, Phys. Rev. Lett. 62, 1067 (1989).

[9] W. Janke and A. M. J. Schakel, Phys. Rev. E 71, 036703 (2005).

[10] C. Vanderzande and A. L. Stella, J. Phys. A 22, L445 (1989).

[11] H. Saleur and B. Duplantier, Phys. Rev. Lett. 58, 2325 (1987).

[12] R. M. Ziff, Phys. Rev. Lett. 56, 545 (1986)

[13] A. L. Stella and C. Vanderzande, Phys. Rev. Lett. 63, 2537 (1989).

[14] B. Duplantier and H. Saleur, Phys. Rev. Lett. 63, 2536 (1989).

[15] T. Grossman and A. Aharony, J. Phys. A 19, L745 (1986).

[16] R. F. Voss, J. Phys. A 17, L373 (1984).

[17] P. Grassberger, J. Phys. A 19, 2675 (1986).

[18] J. L. Cambier and M. Nauenberg, Phys. Rev. B 34, 8071 (1986).

[19] S. M. Allen and J. W. Cahn, Acta Metall. 27, 1085 (1979).

[20] In three dimensions the time variation of the hull enclosed volume is not independent of its size. This can be easily seen by considering a spherical bubble for which $\frac{d V}{d t}=4 \pi R^{2} \frac{d R}{d t}$. In accordance with Eq. (29), $\frac{d R}{d t}=-\frac{\lambda_{h}}{2 \pi} \kappa$, and $\kappa=2 / R$ for a sphere, so, $\frac{d V}{d t}=-4 \lambda_{h} R$. This is the reason why our arguments cannot be extended to higher dimensions.

[21] J. Hoshen and R. Kopelman, Phys. Rev. B 14, 3438 (1976).

[22] See, e.g., A. Weinrib, Phys. Rev. B 26, 1352 (1982).

[23] J. Kalda, Phys. Rev. E 64, 020101(R) (2001), and references therein.

[24] A. D. Rutenberg, Phys. Rev. E 54, R2181 (1996) has shown that lattice anisotropy also induces a very small anisotropy in the coarsening structure.

[25] A. J. Bray, Phys. Rev. Lett. 62, 2841 (1989); A. J. Bray, Phys. Rev. B 41, 6724 (1990); K. Humayun and A. J. Bray, J. Phys. A 24, 1915 (1991).

[26] K. Kaski, M. C. Yalabik, J. D. Gunton, and P. S. Sahni, Phys. Rev. B 28, 5263 (1983).

[27] M. Grant and J. D. Gunton, Phys. Rev. B 28, 5496 (1983); M.-D. Lacasse, M. Grant, and J. Viñals, ibid. 48, 3661 (1993).

[28] K.-t. Leung, J. Phys. A 26, 6691 (1993), and references therein.

[29] H. Hinrichsen and M. Antoni, Phys. Rev. E 57, 2650 (1998).

[30] H. B. Tarko and M. E. Fisher, Phys. Rev. B 11, 1217 (1975).

[31] G. S. Grest and D. J. Srolovitz, Phys. Rev. B 30, 5150 (1984).

[32] M. Fialkowski and R. Holyst, Phys. Rev. E 66, 046121 (2002).

[33] A. E. Jacobs and C. M. Coram, Phys. Rev. B 36, 3844 (1987).

[34] A. Coniglio, J. Phys.: Condens. Matter 13, 9039 (2001).

[35] N. Sator, Phys. Rep. 376, 1 (2003).

[36] A. Sicilia, J. J. Arenzon, A. J. Bray, and L. F. Cugliandolo (unpublished).

[37] C. Chamon and L. F. Cugliandolo, J. Stat. Mech.: Theory Exp. 2007, P07022. 
[38] S. I. Denisov and P. Hänggi, Phys. Rev. E 71, 046137 (2005).

[39] J. G. Amar and F. Family, Phys. Rev. A 41, 3258 (1990); A. J. Bray, J. Phys. A 23, L67 (1990).

[40] B. Derrida and R. Zeitak, Phys. Rev. E 54, 2513 (1996).
[41] P. A. Alemany and D. ben Avraham, Phys. Lett. A 206, 18 (1995).

[42] P. L. Krapivsky and E. Ben-Naim, Phys. Rev. E 56, 3788 (1997). 